\title{
Jogos usados na ReabilitaÇão após ACidente Vascular Cerebral: SCOPIng ReVIEW
}

\section{JUEGOS UTILIZADOS EN REHABILITACIÓN DESPUÉS DE UN ACCIDENTE CEREBROVASCULAR: SCOPING REVIEW}

\section{Games Used In RehabILITATION After StROKE: SCOPING REVIEW}

\author{
DOI 10.33194/rper.2020.v3.n1.4.5740 | Submetido 13/12/2019 | Aprovado 18/05/2020
}

Rita Pires Magalhães ${ }^{2} \mathbb{D}$; Sara Rodrigues Barbeiro' ${ }^{1}$; Vanessa Taveira ${ }^{1} \mathbb{D}$; Bruno Magalhães ${ }^{2,3}$; ; Gustavo Ferreira' ${ }^{1}$ (D)

1 - Centro Hospitalar e Universitário do Porto; 2 - Instituto Português de Oncologia; 3 - Escola Superior de Saúde de Santa Maria

\section{RESUMO}

Objetivos: explorar o estado atual do conhecimento científico relacionado com a utilização de jogos na reabilitação do indivíduo após Acidente Vascular Cerebral, para mapear os jogos e identificar as áreas de função reabilitadas.

Método: revisão do tipo Scoping, com base no modelo do Joanna Briggs Institute. Realizada busca nas bases de dados MEDLINE ${ }^{\circledR}$, CINAHL $^{\circledR}$ e SPORTDiscus ${ }^{\circledR}$. Foram considerados todos os tipos de estudos científicos publicados em inglês, espanhol e português, sem limite temporal.

Resultados: Nos 116 artigos considerados para análise identificaram-se três grandes agrupamentos de jogos: Realidade Virtual imersiva/não imersiva, videojogos e Jogos Tradicionais, que se associaram à reabilitação das funções motora, propriocetiva, cognitiva e cardiorrespiratória.

Conclusão: A utilização de jogos na reabilitação cresceu ao longo dos últimos anos. Os jogos induzem maior interatividade no treino intensivo e repetitivo, acrescentando mais motivação e adesão aos processos de reabilitação, podendo ser um importante recurso terapêutico.

Descritores: AVC; Jogos; Reabilitação

\section{RESUMEN}

Objetivos: explorar el estado actual del conocimiento científico relacionado con el uso de juegos en la rehabilitación de individuos después de un accidente cerebrovascular, para mapear los juegos e identificar áreas de función rehabilitadas.

Método: Revisión del alcance, basado en el modelo del Instituto Joanna Briggs. Busque en las bases de datos MEDLINE $®$, CINAHL $®$ y SPORTDiscus $®$. Se consideraron todos los tipos de estudios científicos publicados en inglés, español y portugués, sin límite de tiempo.

Resultados: En los 116 artículos considerados para el análisis se identificaron tres grandes grupos de juegos: Realidad virtual inmersiva / no inmersiva, Videojuegos y Juegos tradicionales, que se asociaron con la rehabilitación de las funciones motoras, propioceptivas, cognitivas y cardiorrespiratorias.

Conclusión: El uso de juegos en rehabilitación ha crecido en los últimos años. Los juegos inducen una mayor interactividad en el entrenamiento intensivo y repetitivo, agregando más motivación y adherencia a los procesos de rehabilitación, que pueden ser un recurso terapéutico importante.

Palabras clave: Accidente cerebrovascular; Juegos; Rehabilitación

\section{ABSTRACT}

Objectives: To explore the current state of scientific knowledge related to the use of games in the rehabilitation of individuals after stroke, with the objectives of mapping the games and identifying the areas of function rehabilitated.

Method: Scoping review, based on Joanna Briggs Institute model. Search in MEDLINE $®$, CINAHL $₫$ and SPORTDisCus $®$ databases. All types of scientific studies published in English, Spanish and Portuguese, without time limit, were considered.

Results: In the 116 articles considered for analysis three major groupings of games were identified: Immersive / NonImmersive Virtual Reality, Video Games and Traditional Games, which were associated with the rehabilitation of motor, proprioceptive, cognitive and cardiorespiratory functions.

Conclusion: The use of games in rehabilitation has grown over the last few years. Games induce greater interactivity in intensive and repetitive training, adding more motivation and adherence to rehabilitation processes, which can be an important therapeutic resource.

Descriptors: Stroke; Games; Rehabilitation 


\section{INTRODUÇÃO}

O Acidente Vascular Cerebral (AVC), em todo o mundo, é a terceira principal causa de incapacidade para os adultos. O AVC é uma doença devastadora para os indivíduos e suas famílias, sendo também uma das principais causas de demência e depressão ${ }^{(1-2)}$.

As principais sequelas relacionadas com o AVC são a perda total ou parcial da mobilidade do membro superior (MS) e membro inferior (MI), o comprometimento da fala, a perda de capacidade de memória e raciocínio que prejudicam o desempenho das atividades de vida diária e comprometem a qualidade de vida dos indivíduos(3). A reabilitação dos indivíduos com AVC é um processo desafiador. Para ser eficaz, deve ser precoce, intensiva e repetitiva durante várias semanas após a lesão inicial, o que leva ao desafio de como manter a motivação para as pessoas em terapia. Os jogos podem ser uma estratégia eficaz de abordar o problema de envolvimento na terapia ${ }^{(4)}$.

Os jogos são atividades estruturadas com objetivos lúdicos, no entanto são também utilizados e explorados em diferentes contextos, visando aproveitar a sua capacidade para estimular funções cognitivas e físicas. 0 exercício realizado através de jogos permite 0 movimento ativo das áreas afetadas ativando os sistemas sensoriais e cognitivos, beneficiando os indivíduos com $\mathrm{AVC}^{(5)}$. A utilização dos jogos permite desenvolver as potencialidades e diminuir as limitações, procurando estimular as habilidades físicas, mentais e sensoriais, visando a melhoria da qualidade de vida(6).

Uma pesquisa preliminar realizada no Joanna Briggs Institute (JBI) Database of Systematic Reviews and Implementation Reports, Cochrane Library, MEDLINE e CINAHL, revelou que se desconhece a publicação de um mapeamento ou revisão do tipo scoping nesta área.

Conhecedores da importância dos aspetos referenciados, esta revisão pretende explorar o estado atual do conhecimento científico relacionado com a utilização de jogos na reabilitação do individuo após AVC, com o objetivo principal de mapear os jogos usados na reabilitação após AVC. Como objetivo secundário, pretendemos identificar as áreas de função reabilitadas. Ao longo do artigo pretende-se dar resposta às seguintes questões: que tipos de jogos são usados para reabilitar o indivíduo com AVC? Que funções do indivíduo podem ser reabilitadas através dos jogos?

\section{MÉTODO}

Para a concretização dos objetivos, foi elaborada uma scoping review da literatura científica, tendo por base as orientações preconizadas pelo Instituto Joanna Briggs para este tipo de revisão(7). Uma scoping review assume como principais objetivos: formular uma questão inicial ampla e aberta, mapear as evidências existentes subjacentes a uma área de pesquisa, identificar lacunas na evidência existente, constituir um exercício preliminar que justifique e informe a realização de uma revisão sistemática da literatura ${ }^{(8)}$.
Optou-se por este tipo específico de revisão por este ser adequado para determinar o corpo de conhecimento de um determinado tópico e evidenciar o volume de literatura e estudos disponíveis, dando uma visão global do seu conteúdo(9).

Recorreu-se ao modelo PRISMA - Preferred Reporting Items for Systematic Reviews and Meta-Analyses para a organização da informação e foram ainda seguidas as recomendações descritas no PRISMA-SCR (PRISMA Extension for Scoping Reviews) para a apresentação deste relatório ${ }^{(10)}$.

A formulação da questão de investigação baseou-se no acrónimo PCC (População, Conceito e Contexto). Assim, como população foram considerados todos os indivíduos adultos (> 18 anos) com diagnóstico de AVC; para o conceito, a utilização ou recurso a jogos e para o contexto, foi considerada a reabilitação.

Com base na questão norteadora, foram identificados os respetivos descritores em inglês, recorrendo a sintaxes de pesquisa ajustadas a cada uma das bases de dados: MEDLINE ${ }^{\circledR}$ (Medical Literature Analysis and Retrieval System Online), $\mathrm{CINAHL}^{\circledR}$ (Cumulative Index to Nursing and Allied Health Literature) e SPORTDiscus ${ }^{\circledR}$. Foram utilizadas combinações de descritores/medical subject headings (MeSH), subject headings e subjects terms, para cada uma das bases de dados, com recurso a termos livres. Desta forma construíram-se para a pesquisa as seguintes frases booleanas: MEDLINE ${ }^{\circledR}$ - (MH "Stroke+") AND (MH "Video Games") OR (MH "Games, Recreational") OR (MH "Games, Experimental+") AND (MH "Rehabilitation+"); CINAHL ${ }^{\circledR}$ - (MH "Stroke+") AND (MH "Games+") OR (MH "Video Games+") AND (MH "Rehabilitation+"); SPORTDiscus - ((stroke AND games AND rehabilitation) NOT (child OR children)).

Nos critérios de inclusão consideramos todos os artigos elegíveis desde que se identificasse, em indivíduos com AVC, a utilização de jogos na reabilitação. A metodologia de pesquisa não foi restringida a qualquer tipo de estudo primário, sendo incluídos todos os tipos de estudos científicos publicados em inglês, espanhol e português, sem limite temporal na busca. Os critérios de exclusão foram estudos realizados com crianças.

A seleção dos artigos incluídos nesta revisão da literatura passou por um processo rigoroso e sistematizado, apresentado no diagrama de Prisma ${ }^{\circledR}$ que exibe o processo de busca, de exclusão e de seleção dos artigos encontrados. Este processo foi realizado pelos investigadores de forma independente e o resultado final foi obtido após reunião de consenso. Os dados foram obtidos utilizando formulários de extração personalizados para o efeito. Para cada estudo foram documentadas informações sobre autoria, ano de publicação e país; desenho metodológico; características dos jogos e área de intervenção na reabilitação. Posteriormente estes dados foram compilados em quadros e figuras, através de um resumo narrativo dos dados extraídos em cada um dos artigos. 


\section{RESULTADOS}

Pela pesquisa científica realizada nas diferentes bases de dados, obtiveram-se 228 artigos. Após as diferentes etapas apresentadas na Figura 1 foram selecionados 128 artigos para leitura integral dos quais, 116 foram incluídos nesta revisão.

No Quadro 1 são apresentadas as informações resumo dos estudos incluídos. As publicações descritas com jogos ocorrem entre 2002 e 2018, evidenciando um aumento de produção científica nos últimos anos. Em relação à metodologia, destacam-se maioritariamente estudos experimentais, tendo sido identificadas duas revisões sistemáticas. Quanto aos jogos, foi possível identificar três grandes agrupamentos: Jogos de Realidade Virtual imersiva e não imersiva, videojogos e Jogos Tradicionais, e respetivos dispositivos e recursos associados, que se encontram esquematizados na Figura 2.

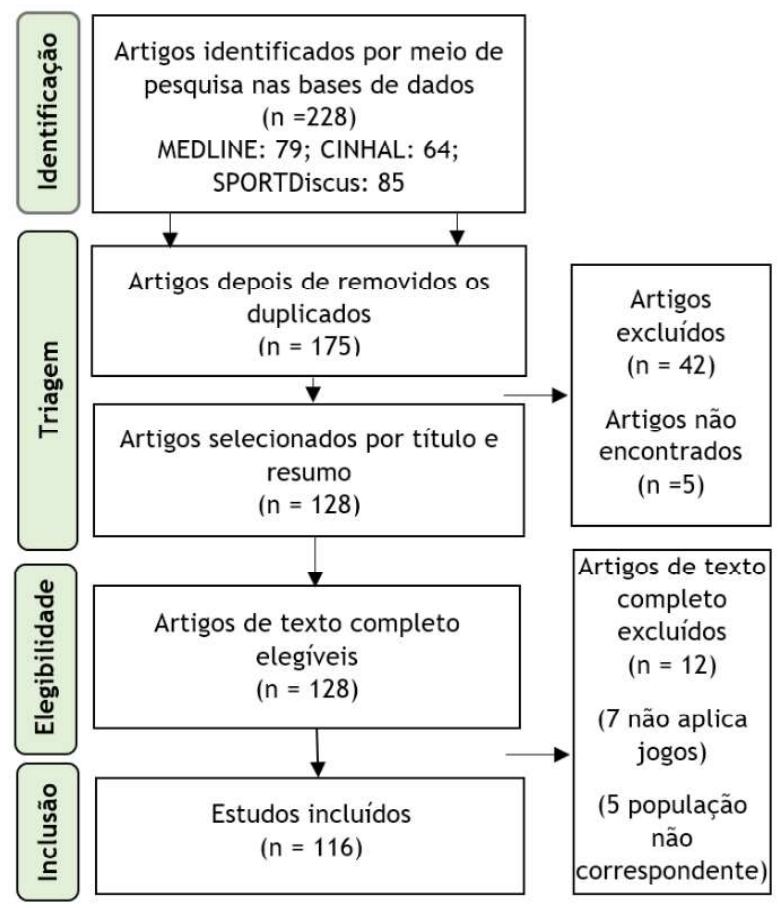

Figura 1 - Fluxograma PRISMA (adaptado) do processo de seleção dos estudos

\begin{tabular}{l|l|l}
\multicolumn{1}{c}{$\begin{array}{c}\text { Estudo } \\
\text { (Autor, País, Ano) }\end{array}$} & $\begin{array}{c}\text { Tipo de } \\
\text { Estudo }\end{array}$ & \multicolumn{1}{c}{ Tipo de jogo/ descrição do jogo } \\
\hline $\begin{array}{l}\text { Bailey, M J. et al., } \\
\text { Reino Unido, 2002 }\end{array}$ & $\begin{array}{l}\text { Estudo } \\
\text { experimental }\end{array}$ & $\begin{array}{l}\text { Jogos de tabuleiro (Snakes and Ladders, Scrabble, Dominó, quebra-cabeça } \\
\text { de palavras). }\end{array}$ \\
\hline $\begin{array}{l}\text { Wood, S.R., et al., } \\
\text { México, 2003(34) }\end{array}$ & $\begin{array}{l}\text { Estudo } \\
\text { experimental }\end{array}$ & $\begin{array}{l}\text { Jogo de computador Pong com dispositivo “Palanca" - alavanca colocada } \\
\text { numa caixa denominada por “carro". Com sensores. Quando necessário } \\
\text { utilizavam luva para segurar o membro afetado à alavanca. }\end{array}$ \\
\hline $\begin{array}{l}\text { Broeren, J., et al., } \\
\text { EUA, 2004(35) }\end{array}$ & $\begin{array}{l}\text { Estudo de } \\
\text { caso }\end{array}$ & $\begin{array}{l}\text { Jogo de RV 3D por computador com dispositivo háptico - estilete portátil. } \\
\text { Consiste em derrubar uma parede de tijolos com uma bola com várias } \\
\text { velocidades. }\end{array}$ \\
\hline $\begin{array}{l}\text { Bekter, A.L., et al., } \\
\text { EUA, 2006(15) }\end{array}$ & $\begin{array}{l}\text { Estudo de } \\
\text { caso }\end{array}$ & $\begin{array}{l}\text { Jogo de computador com tapete de pressão - Under Pressure, Memory } \\
\text { Match, Tic-Tac-Toe (apanhar maças com um cesto). }\end{array}$ \\
\hline $\begin{array}{l}\text { Broeren, J., et al., } \\
\text { Suécia, 2007 }\end{array}$ & $\begin{array}{l}\text { Estudo de } \\
\text { caso }\end{array}$ & $\begin{array}{l}\text { Jogo RV por computador com dispositivo háptico (estilete portátil) que } \\
\text { consiste em derrubar uma parede de blocos com uma bola. }\end{array}$
\end{tabular}

Flynn, S., et al., EUA, 2007(36)

\section{Estudo de caso}

Santos, M.C. México, 2007 (37)

Estudo Jogo de RV com Sony PlayStation 2 Eyetoy (23 Jogos). experimental restritiva e eletroestimulação).

Broeren, J., et al., Suécia, 2008(38)

Estudo comparativo Jogos de RV (Space Tennis, Bingo, Simon, Archery, MugMasterMind e

Broeren, J., et al., Suécia, 2008(39) óculos 3D estereoscópicos.

Rand, D., et al. Israel, 2008(40) Szturm, T., et al., Canadá, 2008(41) Yavuzer, G., et al., Turquia, 2008 ${ }^{(42)}$

Lange, B., et al., EUA 2009(43)
Estudo

\section{experimental} Jogos 3D: Archery; Bingo; Memory; Simon; Space Tennis e Fish como Estudo Jogos de RV: Space Tennis, Wishy-Washy and Kung-Foo em Sony experimental PlayStation Eyetoy.

Estudo Vídeo Jogo interativo com sensor de movimento associado a objetos experimental terapêuticos (bolas, cilindros, etc.) que auxiliam nos jogos.

Estudo Jogos de RV em Playstation EyeToy "Kun-Foo, Goal.Attack, MrChef, Dig experimental $\begin{aligned} & \text { Home-run" combinado com um programa convencional de reabilitação, } \\ & \text { em comparação com reabilitação convencional e visualização do jogo. }\end{aligned}$ EyeToy: jogos boxe e futebol; Wii: jogo golfe e bowling com avatar na tela ou vídeo que capta imagem do paciente.

Estudo Jogo desenvolvido especificamente para a reabilitação com dispositivo experimental háptico (Novint Falcon) com feedback off-the-shelf - o jogador sente peso, forma, textura, dimensão e força de um objecto. Pode ser jogado de forma bimanual.
Áreas de intervenção/ Função

Função motora: MS Heminegligência

Função motora: MS Motricidade fina Lúdica

Função Motora: MS Motricidade Fina

Função Motora

Cognição

Equilíbrio corporal

Função motora

Motricidade fina da

mão

Função motora

Equilíbrio

Proprioceção

Cognição

Função motora

Espasticidade

Função Motora

Motricidade Fina

Cognição

Função Motora: MS Cognição

Função Motora

Lúdico

Função motora: MS Motricidade fina

Função Motora: MS Equilíbrio

Função Motora: MS 


\begin{tabular}{l|l|l}
\multicolumn{1}{c}{$\begin{array}{c}\text { Estudo } \\
\text { (Autor, País, Ano) }\end{array}$} & $\begin{array}{c}\text { Tipo de } \\
\text { Estudo }\end{array}$ & \multicolumn{1}{c}{ Tipo de jogo/ descrição do jogo } \\
\hline $\begin{array}{l}\text { Qiu, Q., et al., } \\
\text { EUA, 2009(44) }\end{array}$ & $\begin{array}{l}\text { Estudo } \\
\text { Experimental }\end{array}$ & $\begin{array}{l}\text { Jogos de RV Imersiva com dispositivo háptico - CyberGloves e CyberGrasp } \\
\text { para os jogos: Plasma Pong, Hummingbird Hunt, Hammer Task, Virtual } \\
\text { Piano. }\end{array}$ \\
\hline $\begin{array}{l}\text { Ruparel, R. et al., } \\
\text { EUA, 2009(45) }\end{array}$ & $\begin{array}{l}\text { Estudo } \\
\text { experimental }\end{array}$ & $\begin{array}{l}\text { Video jogo com sistema TheraDrive que utiliza jogos que simulam } \\
\text { condução (TrackMania, Millipede, Need for Speed) }\end{array}$ \\
\hline $\begin{array}{l}\text { Cameirão, MS., et al., } \\
\text { Espanha, 2010(26) }\end{array}$ & $\begin{array}{l}\text { Estudo } \\
\text { experimental }\end{array}$ & $\begin{array}{l}\text { Sistema de Jogos de Reabilitação baseado em Jogos de RV e } \\
\text { neurorreabilitação. Jogo Spheroids (utiliza um cenário natural semelhante } \\
\text { ànavera, onde interage virtualmente utilizando 2 braços virtuais na } \\
\text { tela). }\end{array}$ \\
\hline
\end{tabular}

Golden, S., et al., Artigo de EUA, 2010 (46) revista

Nintendo Wii sports (futebol e ténis) e Nintendo Wii Fit.

Lange, B., et al.,

EUA, 2010(47)

Saposnik, G., et al., Canadá, 2010(48)

Yong Joo, L., et al., República de

Singapura, 2010(49)

Cameirão, M. S., et

al.,

Espanha, 2011(50)

Hijmans, J.M., et al.,

Nova Zelândia,

2011(51)

Merians, A. S., et al.,

EUA, 2011(52)

Mouawad, M.R., et

al.,

Austrália, 2011(53)

Saposnik, G. and M.

Levin. Canadá, 2011(2)

Schuck, S.O., et al.,

EUA, 2011(54)

Acosta, A.M., et al.,

EUA, 2011(55)

Combs, S.A., et al.,

EUA, 2011(56)

Kim, E. K., et al.,

República da Coreia, 2012 $2^{(57)}$

Hale, L. A., et al., Nova Zelandia, $2012^{(58)}$

Estudo

Jogo de RV com plataforma Nintendo Wii Fit (Balance Board) jogo panda experimental $3 D$ adaptado.

Estudo $\quad$ Jogo de RV Nintendo Wii (Wii Sports and Cooking Mama) Vs

experimenta Terapia Recreativa (cartas de baralho, bingo ou "Jenga")

Estudo Jogos de RV, Nintendo Wii Games Sports com dispositivo Wiimote (boxe, experimental bowling, ténis, golfe e baseball), usado como complemento à terapia convencional.

Estudo Jogo de RV Spheroids através de luvas com sensores de movimento experimental comparando com Reabilitação convencional.

Áreas de intervenção/ Função

Função motora: MS Motricidade fina

Função Motora: MS

Lúdica

Espasticidade

Função Motora: MS

Propriocetiva

Velocidade de resposta

Função Motora

Equilíbrio

Marcha

Equilíbrio

Marcha

Lúdico

Função Motora: MS

Função Motora: MS

Sensorial

Lúdica

Função Motora: MS

Lúdica

Estudo Jogos de RV com computador pessoal (Solitaire, Mah-Jong, FreeCell, and

experimental Bejeweled) semelhantes à Nintendo Wii - com dispositivos CyWee Z,

Handlebar.

studo Jogos de RV imersiva com CyberGlove que usa simulador com jogos

experimental (Plasma Pong, Hummingbird Hunt, Hammer Task, Virtual Piano).

Estudo Jogos de RV - Nintendo Wii Sports (tennis, golf, boxing, bowling e

experimental baseball), com dispositivo Wii mote) com treino formal em laboratório e informal em casa.

Revisão

sistemática Revisão sobre diferentes tipos de sistemas e dispositivos de RV imersiva e

com Meta- não imersiva, usados na neurorreabilitação do MS.

análise

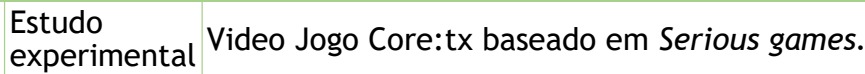

Estudo Jogo de RV Air Hockey 3D videogame, utiliza avatar do braço/interface experimental háptico. \begin{tabular}{l|l} 
Estudo & Jogo- Hand dance pro - com comando adaptado e sensores de movimento.
\end{tabular}

Estudo Wii sports com dispositivo wii remote e Nunchuk controllers (com ajuda experimental de bandas para segurar os comandos caso necessário)

Vs exercícios convencionais e estimulação elétrica do tibial anterior.

Estudo piloto puzzles

Jogos easy-to-see objects and icons, basic sports concepts, or simple

qualitativo com comando controlador de movimentos CyWee Z.

Kam, N.,

Israel, 2012(59)

Estudo piloto Jogos Nintendo: Wii-Sports (Bowling e Ténis); Wii-Fit (Ski-Slalom)

Kim, I.,

Jogos de grupo com brinquedos infantis

Coreia do Sul, 2012 ${ }^{(60)}$ experimental - jogo de futebol

- jogo de golfe.

King M., et al.,

Nova Zelândia,

2012(61)

Estudo

Jogos com comando controlador de movimento CyWee Z:

experimental Popping"; "Mosquito Swat"; "Músic Catch "e" ReBounce ". Jogos desportivos " Air Hockey ".

Jogos de quebra-cabeça "Mah-Jong" e "Solitaire".

Reinthal, A., et al., USA, 2012 ${ }^{(62)}$

Taheri, H., et al., USA, 2012 ${ }^{(63)}$
Jogos selecionados através de um algoritmo que considera várias

Estudo piloto particularidades e características de cada indivíduo: PlayStation II Com EyeToy e NintendoWii (Wii Sports, Wii Resort, e Wii Play).

Estudo Jogo tipo GuitarHero assistido por dispositivo robótico e feed-back visual. Função motora: MS experimental

Função Motora: MS Motricidade Fina
Função Motora: MS

Função Motora: MS Motricidade Fina

Função Motora: MS

Função Motora: MS

Cognição

Reorganização Cortical

Espasticidade

Função Motora: MS

Qualidade de vida

Função Motora: MS

Função Motora: MS com restrição de tronco

Função Motora

Independência

funcional

Equilíbrio

Funcão motora: MS

Equilíbrio

Concentração

Função Motora: MS

Função Motora

Cognição

(fadiga, distúrbio de

sono e depressão)

Função Motora: MS 


\section{Estudo} (Autor, País, Ano)

Finley, M. and S. Combs, EUA, 2012(64)

Fritz, S.L., et al. EUA, $2013^{(65)}$

Gyuchang, L., Républica da Coreia, $2013^{(66)}$

Orihuela-Espina, F., et al.,

México, 2013(27)

Peters, D.M., et al., EUA, 2013(67)

Sin, HH. and Lee, GC.

República da Coreia, 2013(31)

Singh, D.K.A., et al., Malásia, 2013(32)

Tipo de Estudo

Estudo Tipo de jogo/ descrição do jogo

experimental por uma combinação de pistas auditivas via música e pistas visuais através de setas na tela).

Estudo
experimental Xbox Kinect (com terapia ocupacional convencional): Kinect sports (Boxe

Estudo experimental e Bowling); Kinect adventure (Rally Ball, 20,000 Leaks, e Space Pop) vs fisioterapia Convencional. Jogos de RV, baseada na plataforma Gesture Therapy (GT).

Estudo O conjunto de jogos utilizado é o do sistema Armeo (Hocoma, Suíça), com

Descritivo jogos representativos das atividades de vida diária. Para interagir com o jogo, o paciente segura um módulo de mão (com sensor de pressão) que incorpora uma bola (topo) para exercícios de mão.

Estudo Jogos Nintendo Wii (Wii Fit, Wii Sports com Wii balance board) e Sony

Experimental Playstation 2 (EyeToy play 2 e Kinetic games)

Jogos de RV - Xbox Kinect (bowling, boxe, Rally ball, 20,000 Leaks, Space

experimental Jogos

Nitendo Wii Fit Plus, (Balance Bubble com Balance Board)

Estudo

experimental

terapia convencional.

Sucar,L.E., et al., México, 2013 ${ }^{(17)}$

Estudo Gesture Therapy - plataforma baseada em RV. Utiliza 3 Jogos: Steak,

experimental A webcam rastreia movimentos de mão/ inça e traduz isso em comandos para controlar os jogos.

Wüest, S., et al., Suiça, 2013(68)

Estudo Programa de reabilitação que usa jogos de RV com diferentes cenários de Experimental treino.

Microsoft Kinect e plataforma de força Tymo.

Interatividade anatómica virtual baseada em computador, PreMotor Exercise Games (PEGs) usando a Tecnologia 3D PreMotorSkill. Os

Simmons,C.D., et al. Estudo pacientes visualizam o movimento com a ajuda de um membro virtual EUA, 2013(69)

Experimental simulado por computador (terapia de espelho)

Serious games: Agarrar e girar a chave; ação de dois dedos para agarrar a bola e soltá-la no copo. Abrir a caixa de forma correta; jogo de nove caixas com instrucões de voz ao jogador; jogos de quebra-cabeça; jogo de números simples e jogos de letras simples.

Goodman, N.R., et

al.,

Vídeo jogo sentado, com movimento do tornozelo, assistido por robô

EUA, 2014 ${ }^{(70)}$

Bower, K.J., et al., Australia, 2014 ${ }^{(71)}$

Donoso Brown, E.V., et al., EUA 2014 (72)

Friedman, N., et al., EUA, 2014 ${ }^{(73)}$

Hung, J.-W., et al., Taiwan, 2014(74)

Jordan, K., et al., EUA, 2014(24)

Kafri, M., et al., EUA, 2014 ${ }^{(75)}$

Morone, G., et al., Itália, 2014(76)

Novak, D., et al., Suiça, 2014(77)

Estudo piloto (anklebot) para controlar um cursor.

experimental Dispositivo auxiliar: Robô de tornozelo com sensores (que se necessário, pode auxiliar o movimento) com monitorização eletroencefalográfica.

Estudo $\quad$ Jogos de RV da Nintendo - 'Wii Fit Plus' e Wii Sports / Wii Sports Resort (boxe, ciclismo, bowling, arco).

Estudo Vídeo jogo em Laptop - Neurogame Therapy - jogo Peggle, assistido por experimental dispositivo de eletromiografia.

Estudo Terapia com música: uso da "Musicglove" vs terapia convencional com

Experimental mãos e treino isométrico de preensão com "IsoTrainer" Jogo: "Frets on Fire", inspirado no" Guitar Hero".

Nintendo Wii Fit Plus ("Table Tilt", "Ski Slalom", "Soccer Heading",

Estudo "Balance Bubble", "Penguin Slide", "Basic Step" $e$ "Warrior") vs Treino

experimental convencional (exercícios de deslocamento de peso e uso de "rocker board").

Estudo Jogos de computador com dispositivo Smart Skate - tala de antebraço experimental com rato incorporado).

Estudo NintendoWii: Boxing, Run e Penguin experimental Xbox + Kinect: Boxing.

Áreas de intervenção/ Função

Motora: MS

Cognição

Lúdica/motivacional

Função Motora

Equilíbrio

Marcha

Função Motora: MS

Atividades da vida

diária

Função Motora: MS

Motricidade fina

Função Motora

Equilíbrio

Função Motora: MS

Motricidade fina

Função Motora: MI

Equilíbrio

Marcha

Função motora: MS

Motricidade fina

Função motora

Equilíbrio

Marcha

Função motora: MS

Motricidade fina

Cognição

Função motora:

tornozelo

Marcha

Função Motora

Equilíbrio

Função Motora: MS

Motricidade fina

Equilíbrio

Função Motora: MS

Função motora: MS

Equilíbrio

Marcha

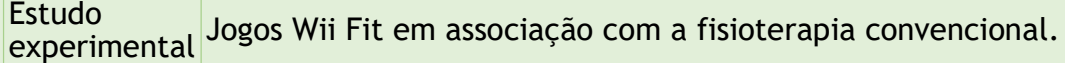

Equilíbrio

Estudo Jogo Air-hockey exibido em ecrã de computador, usando robôs de experimental reabilitação de braço ARMin III.

Função motora: MS 


\begin{tabular}{c|c} 
Estudo & $\begin{array}{c}\text { Tipo de } \\
\text { Estudo }\end{array}$ \\
\hline (Autor, País, Ano) & E
\end{tabular}

Pompeu, J.E., et al., Revisão Brasil, 2014(78)

Sistemas de RV:

IREX - sistema de reabilitação virtual com serious games ("Bird and Ball"; "Coconut" $e$ "Container"; "Juggler"; "Soccer")

Nintendo Wii Fit; Wii Sports (ténis, golfe, boxe, bowling e basebol).

Jogos com plataforma de Força; óculos 3D ("Pinch", "Reaching", "Ball

Shooting", "Rotation"); Sistemas de projeção de ambiente virtual em frente a uma "esteira" associada ou não a um sistema de suspensão de peso; projeção de cenários virtuais interativos com sensores de movimento câmaras.

Marcha

Equilíbrio

Proprioceção

Função motora: MS

Cognição
Shin, J.-H., et al., República da Coreia, 2014(25)

Shiner, C.T., et al., Australia, 2014(79)

Slijper, A., et al., Suécia, 2014 ${ }^{(18)}$

Subramaniam, S., et

al.,

EUA, 2014(80)

EUA, 2014 ${ }^{(81)}$

Viana, R.T., et al., Brasil, 2014(82)

Mei-Hsiang, C., et al., Taiwan, 2014(83)
Taheri, H., et al.

\section{Estudo} observaciona l

Estudo experimental

Estudo experimenta

Sistema RehabMaster (sistema interativo de RV baseado em tarefas específicas para reabilitação pós-AVC) com os jogos: Underwater fire, Goalkeeper, Bug hunter, and Rollercoaster.

Terapia de Movimento baseada na Wii (Nintendo Wii e Wii Sports) combinada com Bilateral priming (uso de dispositivo para realização de movimentos simétricos espelhados).

Consola de jogos baseada em laptop, controlada por 1 ou 2 manípulos Estudo (aperto cilíndrico transversal) para MS

experimental Jogos: Breakout, Snake, Puzzle bubble, Boxing, Recycling, Trombone. (Ambiente doméstico)

Estudo $\quad$ RV - Uso da Wii Fit (com os jogos, Table tilt, Tightrope, Soccer e Balance

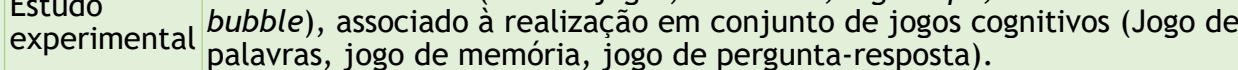

Estudo Vídeo jogo similar ao "Guitar Hero" com dispositivo robótico para a mão experimental FINGER (Finger Individuating Grasp Exercise Robot).

Estudo Estimulação Elétrica Transcraneana combinada com terapia de RV,

Experimental Nintendo Wii ${ }^{T M}$, usando três jogos: "Wii Sports ResortTM", "Wii Play MotionTM" e "Let's TapTM"

Estudo Reabilitação convencional combinada com jogos de RV: nintendo Wii Experimental (bowling e boxe) ou jogos XaviX (bowling e jogos de escalada).
Função motora: MS Equilíbrio

Função motora: MS

Função Motora: MS

Cognição

Equilíbrio

Função motora: MS

(mão e dedos)

Função motora: MS

Função motora: MS

Realidade virtual - jogos (com wiimote Plus), ajustáveis pelo terapeuta,

Tsekleves, E., et al., UK, 2014(84)

Estudo experimental associado a sistema de monitorizaça

Wingham, J., et al., Estudo Nintendo Wii Sports

Reino Unido, 2015(85)

Omiyale, O.,et al., EUA, 2015(86)

experimental - Bowling; Ténis; Basebol e Boxe.

Estudo Nintendo Wii Fit balance board (ski slalom, table tilt, penguin slide, tight experimental rope e balance rope).

Guia de

Anderson, K.R., et al., orientação EUA, 2015(87) clínica

Wii Games, Wii Sports (package): Wii Fit (package); Just Dance (game); Zumba Fitness (game); Wii Ware Arcade Games (package); Kinect Games: Kinect Sports, Bowling, Boxing e Table Tennis; Kinect Adventures.

RV: Jogos interativos de controlo de movimento, 3D: Ball Maze, Fridge

Bower, K.J., et al. Australia, 2015(88)

Estudo Frenzy, Tentacle Dash e Bubble Fish. Utilizam uma câmara com sensor de experimental profundidade (PrimeSense - semelhante á Microsoft Kinect), conectada a laptop.

da Silva Ribeiro, N.M., et al.,

Brasil, 2015(89)

Donoso Brown, E.V., et al.,

EUA, 2015(90)

Hyung Young, L., et al.,

República da Coreia, 2015 (91)

Kyoung-Hee, L., et

al.,

Républica da Coreia, 2015 ${ }^{(92)}$

Estudo RV - Nintendo Wii: Jogos de ténis, hula hoop, futebol e boxe vs experimental fisioterapia convencional.

Estudo

Experimental

NeuroGame Therapy: Jogo Peggle com cursor, que combina biofeedback Qualitativo

$\begin{array}{ll}\text { Estudo } & \text { Programa de treino baseado em RV vs programada de treino orientado } \\ \text { experimental } & \text { tarefa. Treino RV: Uso da Wii fit Plus com a Nintendo Wii, controle }\end{array}$ de eletromiografia de superfície (sEMG). tarefa. Treino RV: Uso da Wii fit Plus com a Nintendo Wii, contro
remoto do Wii, barra de sensor para Wii e Wii Balance Board. 0 movimento é exibido no monitor por um Avatar.

Estudo

Programa de exercícios de RV, que usa o Sistema Interativo de Realidade experimental Virtual com os seguintes jogos: Airborne Rangers, Birds and Balls, Coconuts, Conveyor, Drums, Juggler e Soccer.

Macdonald, J. A. and L. Gauthier.

EUA, 2015(93)
Jogo Virtual 3D e jogo com técnicas comportamentais, que promovem a Estudo resolução de problemas do dia a dia e ativação do membro. Jogo com o experimental rastreamento dos movimentos dos olhos, envolvendo estimulação optocinética e promoção da navegação funcional olhar do lado esquerdo.
Função Motora: MS

Função motora: MS

Função motora: MI

Coordenação

Equilíbrio

Função motora: MS e MI

Equilíbrio

Resistência

Função motora: MS

Cognição

Equilíbrio

Função motora: MS e MI

Equilíbrio

Função Sensorial.

Função motora: MS

(flexores e extensores do punho).

\section{Equilíbrio}

Função motora: MS

Cognição

Desempenho nas

atividades de vida diária

Heminegligência 


\section{Estudo} (Autor, País, Ano)

McNulty, P.A., et al. Austrália, 2015(94)

Mobini, A., et al., Irão, 2015 ${ }^{(95)}$

Paquin, K., et al. Canadá, 2015(20)

Gamito, P., et al., Portugal, 2015(28)

Ponte, S., et al., Itália, 2015(96)

Prange, G.B., et al., Holanda, 2015(97)

Buick, A.R., et al., Irlanda do Norte, 2015(98)

Estudo

Experimental

Estudo Nintendo WiiMote - jogo: Kororinpa Marble Mania

experimental uDraw Game Table: Spongebob, Squigglepants Instant Artist: Studio Alien Splat

Estudo Jogos de RV baseada em Serious games (jogos educacionais) vs grupo sem experimental intervenção.

Microsoft Kinect para Xbox 360 - Jogos:

Estudo Bees and Flowers; Popping Flowers; Colored Cans; Grab your can; experimental Blackboard.

Programa de reabilitação no domicílio.

Jogo de computador, com exercícios interativos 3D, usando um dispositivo de suporte de braço (ArmeoBoom) Vs Exercícios de terapia convencional

Estudo (alcançar objetos posicionados em cima de uma mesa/prateleiras ou experimental empilhar copos, colocar discos, transportar blocos ou estacas, sem o auxílio de suportes mecânicos).

Estação de trabalho ReJoyce (uso de Joystick em jogos de computador de RV) com estimulação elétrica funcional através de uma pulseira. Jogos: corridas de carros, boxe, tiro ao alvo, jardinagem. Jogos baseados em serious games: rodar uma maçaneta, segurar uma lata, rodar uma chave ou desenroscar uma tampa.

Givon, N., et al., Israel, 2015(99)

Estudo Uso de vídeo jogos de RV Vs Exercícios de terapia convencional. Xbox experimental Kinect; Sony Playstation 2 Eyetoy, Sony PlayStation 3; Nintendo Wii Fit, Sistema SeeMe VR Wii Fit walking/jogging game.

Llorens, R., et al., Espanhã, 2015(100)

Bonuzzi, G.M.G., et al., Brasil, 2016(101)

Shin, Ho-Joon., et al., Coreia do Sul, 2016(102)

Estudo
experimental Sistema de posturografia baseado na Wii Balance Board.

Estudo Nintendo Wii com plataforma de equilíbrio.

experimental Jogo: Table Tilt - Wii Fit Plus.

RV combinada com terapia ocupacional - Sistema RAPAEL Smart Glove ${ }^{\mathrm{TM}}$.

Estudo luva com sensor e avatar na tela. experimental espremer laranjas, pescar, cozinhar, limpar o chão, encher copo de vinho, virar páginas de livro.

Nintendo Wii (Wii Sports e Wii Sports Resort) com dispositivo Wiimote -

Kong, K.-H., et al., Singapura, 2016(103)

Estudo jogos: Boxe, bowling, ténis, golfe, beisebol, ténis de mesa, basquete,

experimental ciclismo, disco de frisbee, jogo de espadas e controle de voo em aviões; Vs Terapia convencional.

Lee, M., et al.,

Republica da Coreia, 2016(104)

Mousavi Hondori, H., et al.,

EUA, 2016 ${ }^{(105)}$

$\mathrm{Na}$ Jin, S., et al., EUA, 2016(21)

Estudo Sistema de RV: Microsoft Kinect - exibe o reflexo do utilizador no ecrã ou experimental um marte

Realidade aumentada em vídeo jogo (objetos virtuais projetados pelo

$\begin{array}{ll}\text { Estudo } & \text { computador) - sujeitos vêm o jogo e os seus movimentos de braço } \\ \text { simultaneamente) Vs vídeo jogo em computador pessoal com rato. }\end{array}$ experimental Mesmos movimentos, mas solicitação cognitiva diferente. Jogo Fruit Ninja.

Estudo Jogos de realidade virtual com dispositiva luva - Kinect e P5 Glove experimental (kitchen, archery, puzzle).

Paquin, K., et al., Canadá, 2016 ${ }^{(106)}$

Proffitt, R. and Henderson, W., EUA, 2016(107)

Rinne, P., et al., EUA, 2016 ${ }^{(108)}$

Saposnik, G., et al., Canadá, 2016 (109)

Șimșek, T.T. and K. Çekok, Turquia, 2016(110)
Jogos de realidade virtual: Kororinpa Marble Mania (com WiiMote), experimental Spongebob Squigglepants (com uDraw game tablet), Instant Artist: Studio - Alie Splat (com uDraw game tablet). Estudo de Jogo virtual Mystic Isle através de Kinect.
caso

Estudo Jogo em tablets ou smartphones através de: deslizar com o dedo, toque, experimental joystick, inclinação da tela e um punho adaptado.

Estudo Nintendo Wii vs jogos tradicionais (cartas de baralho, bingo, Jenga ou experimental jogo de bola).

Estudo Nintendo Wii (Wii Sports; Wii Fit) vs conceito Bobath (tratamento experimental convencional de reabilitação neurológica).
Áreas de intervenção/ Função

Função motora: MS

Função motora: MS e tronco.

Motricidade fina

Cognição (memória, concentração, orientação espacial)

Função motora: MS

Função motora: MS

Função motora: MS

Motricidade fina

Função motora: MS e MI Equilíbrio

Marcha

Equilíbrio

Proprioceção

Equilíbrio

Função motora: MS (extremidade distal)

Função motora: MS

Equilíbrio

Motricidade fina

Função motora: MS

Função Motora: MS

Função Motora: MS

Função Motora: MS Cotricidade fina Cognição

Função Motora

Função Motora: MS

Função Motora: MS Motricidade Fina

Motora: MS

Equilíbrio

Marcha

Melhoria das AVD 


\section{Estudo}

(Autor, País, Ano)

Adie, K., et al.,

UK, 2016(19)

Bang, Y., et al., República da Coreia, 2016 (111)

Zondervan, D.K., et al., EUA, $2016^{(112)}$

Khor, K. X., et al., Malásia, 2016(14)

Nijenhuis, S.M., et al., Netherlands, $2016^{(113)}$

Rand, D., et al., Israel, 2016(114)

Standen, P. J., et al. Reino Unido, 2016(115)

Trinh, T., et al., EUA, 2016 ${ }^{(116)}$

Áreas de intervenção/ Função

Estudo Nintendo Wii sports (bowling, tennis, golf, baseball) com controlo remoto experimental vs exercícios de braço no domicílio.

Estudo $\quad$ RV com Nintendo Wii - através de dispositivo wii board. Comparação com experimental grupo que usou treino de marcha em passadeira.

Estudo Exercícios convencionais (exercícios de mesa) vs terapia com "music experimental glove" em laptop - jogo similar ao guitar hero.

Estudo Dispositivo robótico de membro superior, associado a jogo de RV que experimental assiste de forma passiva, ativa-assistida e resistida.

Estudo Jogos de computador com

experimental touchscreen associado a ortótese de punho e mão vs exercícios em livro de exercícios/atividades.

Estudo Xbox Kinect: Bowling, Table Tennis, 20,000 Leaks - para exercícios em pé. experimental Sony PlayStation 2 EyeToy: Wishi washi, Ghosts, Kong fu - para exercícios sentado.

Estudo Vídeo jogos de realidade virtual de baixo custo (uso doméstico) com luva Experimental virtual vs terapia convencional ver jogo: Spacerace Balloonpop Spongeball

Estudo

observaciona Wii sports (golf, basebal, bowling, ténis e boxe). l

Bouchard, A.E., et al., Estudo Jogo Pinball-like com a mão posicionada num dispositivo robótico que experimental induz uma orientação háptica ou uma ampliação do erro.

Canadá, 2016 ${ }^{(117)}$

Choi, H., et al., Républica da Coreia, 2017 (30)

Estudo Wii fit game (ski slalom e soccer heading) associada a Terapia de experimental movimento induzida por restrição vs grupo sem terapia de restrição.

Vídeo jogo com sensores de movimento no MS associado a terapia de al., EUA, 2017(29) experimental movimento induzida por restricão, assistida por terapeuta ou vídeo assistência, vs terapia de restrição convencional vs terapia convencional.

Grossi, M., et al., Brasil, 2017(118)

\section{Estudo Wii fit com balance board (jogos pinguim e corda bamba, jogo das} experimental bolinhas e jogo do rio).

Jogos Wii Fit (Table Tilt, Soccer Heading, Balance Bubble, Penguin Slide,

Hung, J. W., et al., Tailandia, 2017 23 (23)

Estudo e Perfect 10), sistema Tetrax balance biofeedback (Speedtrack, Catch, experimental Skyball, Gotcha, Speedball, Tag, Freeze, Immobilizer) vs treino de equilíbrio convencional.

James, T.T. e

Brammatha, A., India, 2017 (119)

Park, D. -S., et al., República da Coreia, 2017 ${ }^{(120)}$

Rowe, J. B., et al., EUA, 2017(121) Jung, S-M. et al., República da Coreia, 2017(122)

Carregosa, A.A., et al.,

Brasil, 2017 ${ }^{(123)}$

Huang, X., et al., Austrália, 2017(3)

Rand, D., et al., Israel, 2018(22)

Choi, Y.-H. and Paik, N.-J.,

Estudo “Thera - Trainer Balo" (dispositivo robótico semelhante a um standing-

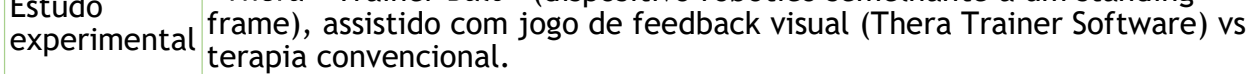

Estudo RV - Xbox Kinect: boxing, table tennis, soccer, golf, ski, football; vs experimental fisioterapia convencional.

\begin{tabular}{l|l} 
Estudo & Dispositivo robótico de dedos que assiste jogo semelhante ao Guitar Hero.
\end{tabular} Estudo
experimental

Função Motora: MS

Equilíbrio

Marcha

Função Motora: MS (mão)

Função Motora: MS (punho e antebraço)

Função Motora: MS Motricidade fina

Função motora: MS Equilíbrio

Função motora: MS

Função motora: MS e MI Equilíbrio

Marcha

Função Motora: MS

Proprioceção

Função Motora: MI

Equilibro

Função Motora: MS

Equilíbrio

Marcha

Equilíbrio

Cognição

Equilíbrio (postura)

Função Motora: MS e MI Equilíbrio

Marcha

Função motora: MS

Motricidade fina

Função motora: MS Desempenho nas AVD

\begin{tabular}{l|l} 
Estudo & Wii sports e Wii fit (Boxe, ténis, futebol e hula hoop).
\end{tabular}

Função motora: MS

Equilíbrio

\begin{tabular}{l|l} 
Estudo & Joxperimental \\
expos de RV assistidos por dispositivo robótico de mão (Amadeo).
\end{tabular}

Função motora: MS (mão)

Motricidade fina

Estudo Jogos com os dispositivos: Microsoft Xbox Kinect, Sony PlayStation 2

Função motora: MS

Eyetoy, Sony PlayStation 3, Nintendo Wii Fit e o sistema SeeMe VR (Virtual Equilíbrio

Reality Kinect Rehabilitation, 2016).

Marcha

Estudo $\quad$ RV através de jogos visualizados em tablet, com recurso a smartphone experimental preso ao antebraço para detetar o movimento.

Função motora: MS

República da Coreia, $2018^{(124)}$

Silva de Sousa, J. C., Estudo

et al., Brasil, 2018 ${ }^{(125)}$ experimental XBox360+Kinect: Jogos de ténis e boxe.

Karasu, A. U., et al., Estudo Nintendo Wii Fit e Wii Balance Board - Jogos: Heading, Ski Slalom, Table Turquia, $2018^{(12)}$ experimental Tilt, Tightrope Tension, Balance Bubble and Penguin Slide; associada a experimental reabilitação convencional vs apenas reabilitação convencional.
Cardiorrespiratório

(Impacto na FC e

consumo de oxigénio)

Equilíbrio 


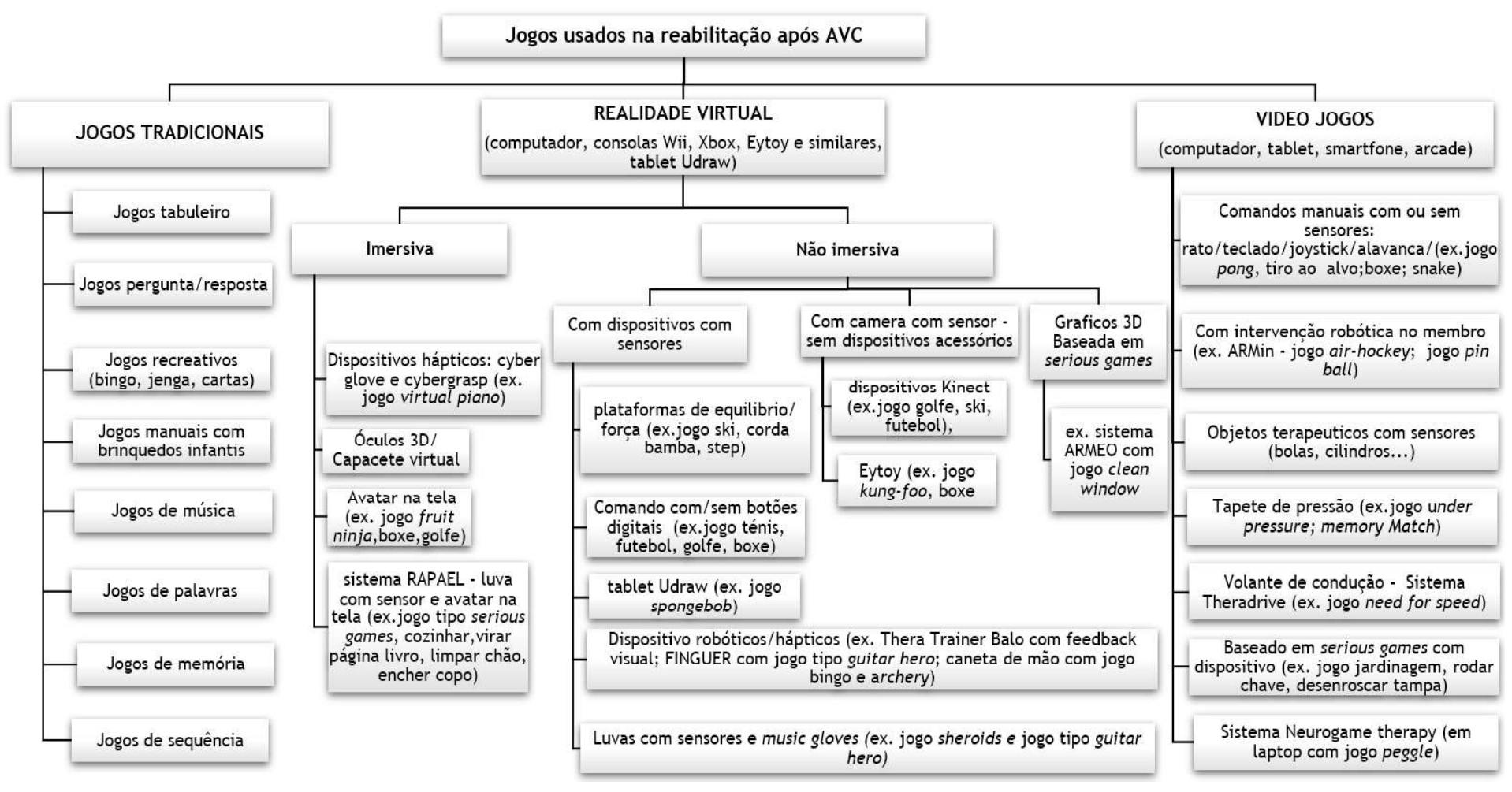

Figura 2 - Tipos de jogos

0 primeiro artigo resultante desta pesquisa data de 2002 e estuda a utilização de jogos tradicionais, através do uso de jogos de tabuleiro, para reabilitação da função motora do membro superior (MS) com heminegligência. Nesta década, apesar de o número de artigos encontrado ser reduzido (menor que 17\% do total de artigos), consegue-se já identificar a utilização dos três grandes agrupamentos de jogos.

Do total de resultados obtidos na pesquisa, os jogos tradicionais surgiram referidos em $7 \%$ dos artigos, os videojogos em $21 \%$ e os jogos de realidade virtual em $72 \%$. Estes últimos surgem em grande número principalmente pela utilização de consolas comerciais (52\%), que começam a ser estudadas na reabilitação em 2007 (no mesmo ano em que são lançadas comercialmente). É neste ano que identificamos o primeiro artigo que estuda a utilização de uma consola comercial (Sony Playstation2 Eyetoy ${ }^{\circledR}$ ) que através de uma câmara com sensor de movimento, capta a imagem do jogador projetando-a na tela e usa os movimentos do corpo para interagir com os objetos virtuais dentro da tela. Posteriormente, outra consola comercial, Nintendo $\mathrm{Wii}^{\circledR}$, que se caracteriza pela utilização de um dispositivo de controle sem fios, o Wii Remote, dotado de um acelerómetro capaz de detetar movimentos em três dimensões, foi identificada num artigo de 2009. A partir desta data multiplicam-se os artigos que referem este tipo de consolas. São referidos também o uso de equipamentos associados a essas consolas, tais como a plataforma de força TYMO ${ }^{\circledR}$ (sistema de posturografia portátil e plataforma de equilíbrio), a Wii Balance Board (uma balança com sensores de pressão que permite que o jogador faça várias atividades físicas, como jogos desportivos ou yoga) e a uDraw GameTablet (tablet gráfico para jogos que possui uma caneta sensível à pressão que permite aos usuários desenhar e visualizar na tela). Para além das consolas comerciais, foram identificados outros tipos de equipamentos associados a videojogos ou jogos de realidade virtual, tais como, computadores (fixos ou portáteis), arcade, tablet e smartphone. Os equipamentos associados aos jogos tradicionais foram jogos de tabuleiro, brinquedos infantis, instrumentos musicais, livros de atividades e mesas de atividades.

Descritos no Quadro 2 estão mencionados os diferentes dispositivos associados aos videojogos e jogos de realidade virtual que permitem uma maior jogabilidade e interação com o jogo, podendo promover também feedback sensorial e/ou motor.

Foram identificados com maior representatividade no final da presente década sistemas de jogos baseados em realidade virtual, desenvolvidos e concebidos especificamente para a reabilitação, de forma a proporcionar um tratamento mais individualizado e a manter o paciente mais motivado. Estes sistemas agregam diferentes jogos em diferentes ambientes virtuais que podem ser personalizados de acordo com as necessidades dos pacientes. Estes sistemas incluem jogos da classe serious games. Apesar de não haver uma definição clara sobre o termo serious games, esta classe de jogos visa principalmente a simulação de situações práticas do dia-a-dia. 0 termo serious games passou a ser utilizado para identificar os jogos cujo propósito é favorecer a absorção de conceitos e habilidades psicomotoras, beneficiando das características lúdicas e recreativas ${ }^{(11)}$.

Através da análise da descrição dos jogos foi possível ainda identificar as diferentes classes de jogos usados na reabilitação: jogos de palavras, jogos recreativos, jogos de memória, pergunta/resposta, estratégia, quebra-cabeças, jogos de música e ritmo, jogos desportivos, de atividade física e de aventura, jogos de habilidade e serious games. 


\section{DISPOSITIVOS}

\section{DESCRIÇÃO}

Com sensores

Plataformas

De suporte

Tipo luvas

robóticos
Comandos (ex: cewezz, wiimote); palanca; objetos terapêuticos bolas e cilindros; tapete de pressão; Thera Drive® (volante para jogo de condução).

Plataforma de força $T Y M O{ }^{\circledR}$ (sistema de posturografia portátil e plataforma de equilíbrio); Wii Balance Board (balança com sensores de pressão que permite que o jogador faça várias atividades físicas, como jogos desportivos ou yoga); esteira de marcha; tapete de pressão.

ArmeoBoom (suporte de braço e mão ajustável, que neutraliza os efeitos da gravidade e facilita movimentos auto-iniciados com maior feedback num ambiente de trabalho tridimensional); Smart Skate (tala de antebraço com rato incorporado).

Frequentemente usados para jogos semelhantes ao Guitar Hero®, nomeadamente na reabilitação da extremidade distal do MS e treino de motricidade fina: Music Glove/P5 Glove/Cybergloves ${ }^{\circledR}$ (luvas com sensores); Cybergrasp (exoesqueleto háptico de mão); FINGER (robô com mecanismos de curvatura dos dedo); Sistema RAPAEL Smart Glove TM (sistema de biofeedback projetado para a reabilitação da extremidade distal do MS do paciente com AVC, através de um dispositivo de luva com sensor que rastreia o movimento e a postura do membro usando um aplicativo de software que permite jogar diferentes jogos, manipulando mãos ou objetos virtuais).

Estilete portátil; caneta de mão; The Phantom Omni® (dispositivo semelhante a caneta que interage com cenário virtual, através de bancada semi-imersiva e óculos 3D estereoscópicos); Novint Falcon (proporciona treino bimanual, permitindo ao jogador sentir peso, forma, textura, dimensão e força de um objecto); robótico de mão em jogo Pinball-like que induz uma orientação háptica ou uma ampliação do erro; robótico de mão Amadeo (assiste de forma passiva ou ativa-assistida a flexão e extensão de cada dedo; robótico de braço ARMin III (fornece até sete graus de liberdade para articulação do ombro e cotovelo e inclui um módulo manual); robô para tornozelo Anklebot (permite realizar flexão dorsal ou flexão plantar do tornozelo parético para mover o cursor na tela); Thera trainer balo (dispositivo semelhante a standing frame, para exercícios de equilíbrio dinâmico e controle postural assistido por feedback visual através de jogo em computador).

Quadro 2 - Dispositivos associados a jogos

SISTEMAS

Rehabilitation Gaming System

SeeMe

Reab Master

Gesture Therapy

IREX®
Proporciona treino de neurorreabilitação para a extremidade do membro superior afetado.

Combina tarefas terapêuticas com jogos interativos (tecnologia Kinect) para tornar o processo de reabilitação mais fácil, divertido e eficaz. Pode praticar e avaliar a força, resistência, amplitude de movimento, controle postural, tempo de reação, propriocepção, qualidade de movimento, perceção, atenção e memória.

Fornece um ambiente rico de reabilitação interativa e usa o conceito de jogos, sem a necessidade de usar algum dispositivo, reproduzindo o movimento através de avatar. Envolve quatro jogos (desportivos, de habilidade e aventura) que solicitam o movimento do membro superior e/ou tronco para treinar o controle, resistência, velocidade, precisão, amplitude de movimento e coordenação mão-visão.

Plataforma/terapia baseada em realidade virtual para reabilitação motora do membro superior que favorece os princípios da reabilitação (repetição, feedback, motivação e treino para tarefa específica), desafiando o paciente a realizar tarefas diárias, em forma de serious games, em ambiente virtual seguro.

Sistema Interativo de Reabilitação e Exercício GestureTek que utiliza a tecnologia de controle imersivo por vídeo, para colocar os pacientes em ambientes virtuais de jogos, onde são guiados por regimes de exercícios terapêuticos.

Quadro 3 - Sistemas de jogos concebidos para a reabilitação.

Terapias associadas à execução de jogos foram referidas em alguns artigos, tais como, a terapia de movimento induzida por restrição, electroestimulação, estimulação elétrica transcraneana ou terapia de espelho, por forma a potenciar a recuperação funcional do indivíduo. Encontrou-se também um videojogo (Peggle) que associa um biofeedback de superfície NeuroGame Therapy, com objetivo de promover a recuperação motora do MS (flexores e extensores de punho).

A maioria dos jogos identificados foram usados com o objetivo de recuperação da função motora do MS (55\%). Outras áreas de intervenção mencionadas foram: equilíbrio, postura, marcha e proprioceção referidos em 23\%; motricidade fina referida em 12\%; cognição em $9 \%$ e foi encontrado apenas um estudo experimental do ano 2018 que investigou o impacto dos jogos (ténis e boxe) na função cardiorrespiratória (impacto na frequência cardíaca e consumo de oxigénio) através da utilização de uma consola comercial.

Um tipo de jogo referido com frequência na reabilitação da função motora das mãos e dedos é o jogo do género Guitar Hero, associado a dispositivos hápticos e robóticos. Para o treino de equilíbrio destacam-se os jogos que utilizam as plataformas de equilíbrio, que permitem jogar jogos da classe de 
atividade física, habilidade, desportivos e aventura, tais como, ski slalom, table tilt, penguin slide, balance rope, soccer heading, balance bubble, basic step. Para o treino cognitivo destacam-se os serious games. $\mathrm{Na}$ reabilitação dos MS e MI destacam-se os jogos classificados como desportivos, presentes nos três grandes agrupamentos de jogos (jogos tradicionais, videojogos e realidade virtual), tais como, futebol, boxe, bowling, ténis, golfe, basebol.

\section{DISCUSSÃO}

Os jogos de realidade virtual são os que têm despertado mais interesse na investigação sobre o seu uso na reabilitação após AVC, tendo sido de forma inequívoca os mais encontrados nesta revisão. Nos últimos anos, houve um crescente interesse no uso de novas tecnologias, como a realidade virtual em reabilitação de indivíduos após AVC. Os resultados clínicos indicam que o uso de tecnologias de realidade virtual melhora o funcionamento motor, podendo ser usadas para melhorar a função do membro superior, a marcha e equilíbrio, a função motora global e a função cognitiva em pacientes com AVC (12). Por outro lado, os jogos tradicionais foram os menos identificados, sendo que a maioria foi estudada em associação ou comparação com videojogos e jogos de realidade virtual.

Os videojogos e jogos de realidade virtual são muitas vezes referidos com recurso a dispositivos com sensores hápticos ou robóticos. Os robôs de reabilitação tornaram-se ferramentas importantes na reabilitação do indivíduo com AVC. Comparado ao treino manual do braço, o treino suportado por robô pode ser mais intensivo, de maior duração e mais repetitivo, conferindo aos robôs o potencial de melhorar o processo de reabilitação em pacientes com AVC ${ }^{(13)}$. No entanto, o custo dos robôs de reabilitação ainda é um problema, limitando o seu perfil de custo-benefício e dificultando a avaliação e implementação em larga escala ${ }^{14}$. Os dispositivos hápticos, identificados num elevado número de artigos com o objetivo de potenciar a função do membro afetado, podem exercer o movimento de forma passiva, ativa assistida ou resistida, com a possibilidade de transmitirem um feedback a vários níveis, desde vibrotátil, visual, auditivo ou mesmo propriocetivo. A utilização destes dispositivos tem sido sugerida para melhorar a reabilitação do indivíduo com AVC, usando a realidade virtual de forma a aumentar a motivação dos utilizadores $^{(15-16)}$. O feedback melhora a aprendizagem, sendo de extrema importância para a aprendizagem motora. Evoca processos neurofisiológicos que induzem profundas alterações corticais e subcorticais. Em geral, os jogos em computador são excelentes em fornecer feedback, contribuindo ainda mais para manter o jogador envolvido ${ }^{(17)}$.

A realidade virtual pode ser classificada de não imersiva a totalmente imersiva, dependendo do grau em que o usuário está isolado do ambiente físico envolvente quando interage com o ambiente virtual. A realidade virtual imersiva não é tão utilizada em estudos experimentais, pois é mais cara e implica a sua utilização de forma controlada em laboratório(2). Os jogos mais encontrados na nossa amostra foram os de realidade virtual não imersiva associados a dispositivos comerciais, tais como, a Nintendo Wii e outros semelhantes. A indústria do entretenimento desenvolveu estes jogos para uso doméstico, tornando esta tecnologia menos dispendiosa e de mais fácil acesso para o seu uso na reabilitação ${ }^{(12)}$. Vários desses jogos foram adotados por profissionais de saúde como intervenções de reabilitação, embora não tenham sido inicialmente projetados para esse efeito. A interatividade que caracteriza estes jogos, bem como os diferentes dispositivos associados, permitem tornar o ambiente de jogo mais real, mais dinâmico, desafiante, competitivo, estimulante e motivante para o utilizador. A motivação do paciente é fundamental para exercer a adesão ao exercício ${ }^{(17)}$. O desafio e a concorrência inerentes aos jogos são elementos que a podem melhorar. Considerando que para a reabilitação do individuo após AVC são fatores importantes a repetição, o feedback, o treino orientado à tarefa e a motivação, estes jogos são uma importante ferramenta para aumentar a adesão(17-18) e podem motivar os pacientes na interligação com amigos e familiares, dando-lhes uma sensação social de conexão(17).

Pela cronologia dos estudos publicados, foi possível constatar uma evolução na utilização dos jogos que acompanha o desenvolvimento tecnológico. Diferentes tipos de dispositivos foram surgindo de forma a tornar a envolvência do utilizador cada vez maior, utilizando dispositivos fáceis de manipular, com a possibilidade de transmitirem um feedback a vários níveis em simultâneo. Foram encontrados sobretudo para o membro superior (ex. dispositivo Wii Remote) ${ }^{(19)}$ e sua extremidade distal (ex. tablet udraw) ${ }^{(20)}$; P5 Glove ${ }^{(21)}$. Também para o tronco e membros inferiores foram investigados dispositivos, tais como, o Thera Trainer Balo, dispositivo que se assemelha a um standing frame (22-23) para permitir ao utilizador jogar interactivamente jogos de realidade virtual (com feedback visual), realizando simultaneamente treino de postura e equilíbrio, sendo adequado para pacientes incapazes de permanecer em pé ou com elevado risco de queda, proporcionando uma forma de exercício eficaz e segura.

O recurso a ortóteses de braço e mão ou suportes para o membro (exemplo: arm skate) ${ }^{(24)}$, foram também referidos em vários artigos como estratégia fundamental para o utilizador conseguir usar os comandos necessários para a realização do jogo com o seu membro afetado.

Foram encontrados nesta revisão, principalmente nos anos mais recentes, sistemas de jogos baseados em realidade virtual, desenvolvidos especificamente para a reabilitação. Estas plataformas de realidade virtual, capazes de auxiliar o paciente no treino, apoiam-se nos princípios da reabilitação promovendo repetição, treino orientado à tarefa, feedback apropriado e meio ambiente motivante. Estes sistemas usam conceitos idênticos aos jogos comerciais, dos quais se destacam várias vantagens: baixo custo, um ambiente atrativo, protocolos de treino gradual e individual, ampla adaptabilidade às necessidades e progresso do paciente, recursos para gravação de desempenho, 
produzindo feedback em tempo real para paciente e terapeuta, e a possibilidade de ser usado com supervisão mínima do profissional de saúde, facilitando a prescrição domiciliar. O sistema RehabMaster provou ser viável e seguro para melhorar a função da extremidade superior em pacientes com AVC(25). Os jogos usados nestes sistemas são, maioritariamente, da classe serious games simulando atividades de vida diária(26-27). Estes contêm um conteúdo educacional que contrasta com os jogos de computador tradicionais, cujo objetivo principal é entreter. Os serious games incluem jogos de aprendizagem, jogos para a saúde e jogos para políticas e mudanças sociais, daí o sector da saúde demonstrar um maior interesse nestes jogos ${ }^{(28)}$.

Associados aos jogos, foram identificados artigos onde se tentou potenciar o efeito do jogo, associando a sua execução a técnicas de terapia convencional, tais como, o movimento em espelho ou a terapia do movimento induzida por restrição. Sabe-se que a restrição mecânica e funcional aumenta o uso do membro mais afetado e promove a plasticidade cerebral(29), bem como, recupera a simetria dos membros inferiores melhorando o equilíbrio( ${ }^{(30)}$.

O uso de jogos - quer associado à terapia convencional, quer como grupo de comparação - permitiu concluir a alguns autores que a sua utilização no treino orientado para exercícios com a extremidade parética (repetição, intensidade) permite obter os mesmos resultados da reabilitação convencional, num menor período de tempo ${ }^{31}$. Possibilita também diminuir o tempo despendido pelo terapeuta com os indivíduos ${ }^{32}$ e aumenta a motivação e a adesão ao tratamento.

\section{CONCLUSÃO}

O objetivo desta scoping review foi mapear e analisar os jogos usados na reabilitação dos indivíduos após AVC. Foram obtidos 116 estudos onde foi possível identificar três grandes agrupamentos de jogos: Jogos de Realidade Virtual imersiva e não imersiva, videojogos e Jogos Tradicionais. Estes jogos foram usados para a reabilitação da função motora global e da função cognitiva em pacientes com AVC, sendo que a maioria dos jogos identificados foram usados para a recuperação da função motora do MS.

A utilização de jogos nos processos de reabilitação dos indivíduos tem vindo a crescer ao longo dos últimos anos, acompanhando o desenvolvimento tecnológico e tornando este processo mais interativo, motivante e estimulante para o utilizador. 0 treino intensivo e repetitivo, aliado à interatividade e motivação que thes estão inerentes, facilita a adesão à reabilitação, podendo melhorar a sua eficácia. Os jogos de realidade virtual são os que têm despertado mais interesse.

O conhecimento sobre a utilização de jogos e o seu desenvolvimento poderão exercer um valioso contributo para os processos de recuperação dos indivíduos após AVC e para os respetivos profissionais de saúde, devendo ser cada vez mais uma ferramenta a considerar. Desta forma, consideramos que no futuro se poderá completar o tema deste estudo com uma investigação mais aprofundada sobre as funções que poderão ser reabilitadas com cada tipo de jogo e sobre os contextos ambientais onde se utilizam os jogos para a reabilitação do individuo após o AVC. Concluímos assim que os resultados desta revisão poderão ser úteis tanto para a prática clínica no contexto da Enfermagem de Reabilitação como também para futuras investigações na área.

Algumas limitações encontradas neste estudo relacionaram-se: com a descrição pouco pormenorizada dos jogos que alguns autores referem; com os conceitos associados à realidade virtual e sua imersividade que nem sempre se encontram bem esclarecidos $e$ apresentam diferentes interpretações; e a falta de clareza por vezes encontrada na descrição do uso de jogos tradicionais na reabilitação convencional.

\section{REFERÊNCIAS BIBLIOGRÁFICAS}

1. Johnson W, Onuma O, Sachdev S. Stroke: a global response is needed. 2016;

2. Saposnik G, Levin M. Virtual Reality in Stroke Rehabilitation A Meta-Analysis and Implications for Clinicians. 2011;1380-6.

3. Huang X, Naghdy F, Naghdy G, Du H, Todd C. The Combined Effects of Adaptive Control and Virtual Reality on Robot-Assisted Fine Hand Motion Rehabilitation in Chronic Stroke Patients : A Case Study. J Stroke Cerebrovasc Dis [Internet]. 2017;1-8. Available from: https://doi.org/10.1016/j.jstrokecerebrovasdis.2017.08.027

4. Burke JW, McNeill MDJ, Charles DK, Morrow PJ. Serious Games for Upper Limb Rehabilitation Following Stroke. 2009;

5. Proffitt R, Otr L, Lange B, Hons B. Feasibility of a Customized, InHome, Game-Based stroke exercise program using The Microsoft Kinect ${ }^{\circledR}$ Sensor. Int Journal Telerehabilitation. 2015;7(2)

6. Bittencourt, J R., Walter E. Workshop de Jogos Digitais na Educação. Ludens Art. Gravataí; 2006.

7. The Joanna Briggs Institute Reviewers 'Manual 2015 Methodology for JBI Scoping Reviews. 2015;

8. Peters MDJ, Godfrey CM, Khalil H, Mclnerney P, Parker D SC. Guidance for conducting systematic scoping reviews. 2015;

9. Munn Z, Peters MDJ, Stern C, Tufanaru C, McArthur A AE. Systematic review or scoping review? Guidance for authors when choosing between a systematic or scoping review approach. Med Res Methodol. 20018;18(1):143.

10. Liberati A, Altman DG, Tetzlaff J, Mulrow C, Gøtzsche PC, loannidis JPA, et al. The PRISMA Statement for Reporting Systematic Reviews and Meta-Analyses of Studies That Evaluate Health Care Interventions : Explanation and Elaboration. J Clin Epidemiol. 2009;62(10):e1-34.

11. Blackman S. Serious games... and less!. ACM Siggraph Comput Graph. 2005 Feb 1;39(1):12-6.

12. Karasu AU, Batur EB. Effectiveness of Wii-based rehabilitation in stroke: a randomized controlled study. J Rehabil Med. 2018 May 5;50(5):406-12.

13. Nef T, Guidali M, Riener R. ARMin III-arm therapy exoskeleton with an ergonomic shoulder actuation. Appl Bionics Biomech. 2009 Jan $1 ; 6(2): 127-42$.

14. Khor KX, Chin PJ, Yeong CF, Su EL, Narayanan AL, Rahman HA, Khan QI. Portable and reconfigurable wrist robot improves hand function for post-stroke subjects. IEEE Trans Neural Syst Rehabil Eng. 2017 Apr 7;25(10):1864-73.

15. Betker AL, Szturm T, Moussavi ZK, Nett C. Video game-based exercises for balance rehabilitation: a single-subject design. Arch Phys Med Rehabil. 2006 Aug 1;87(8):1141-9.

16. Broeren J, Rydmark M, Björkdahl A, Sunnerhagen KS. Assessment and training in a 3-dimensional virtual environment with haptics: a report on 5 cases of motor rehabilitation in the chronic stage after stroke. Neurorehabil Neural Repair. 2007 Mar;21(2):180-9.

17. Sucar LE, Orihuela-Espina F, Velazquez RL, Reinkensmeyer DJ, Leder R, Hernández-Franco J. Gesture therapy: An upper limb virtual reality-based motor rehabilitation platform. IEEE Trans Neural Syst Rehabil Eng. 2013 Dec 5;22(3):634-43. 
18. Slijper A, Svensson KE, Backlund $P$, Engström $H$, Sunnerhagen KS. Computer game-based upper extremity training in the home environment in stroke persons: a single subject design. J Neuroeng Rehabil. 2014 Dec 1;11(1):35.

19. Adie K, Schofield C, Berrow M, Wingham J, Humfryes J, Pritchard C, James M, Allison R. Does the use of Nintendo Wii SportsTM improve arm function? Trial of WiiTM in Stroke: A randomized controlled trial and economics analysis. Clin Rehabil. 2017 Feb;31(2):173-85.

20. Paquin K, Ali S, Carr K, Crawley J, Mcgowan C, Horton S. Effectiveness of commercial video gaming on fine motor control in chronic stroke within community-level rehabilitation. Disabil Rehabil [Internet]. 2015;0(0):1-8. Available from: http://dx.doi.org/10.3109/09638288.2014.1002574

21. Seo NJ, Kumar JA, Hur P, Crocher V, Motawar B, Lakshminarayanan K. Usability evaluation of low-cost virtual reality hand and arm rehabilitation games. J Rehabil Res Dev. 2016;53(3):321-34.

22. Rand D, Givon N, Avrech Bar M. A video-game group intervention: Experiences and perceptions of adults with chronic stroke and their therapists. Can J Occup Ther. 2018 Apr;85(2):158-68.

23. Hung JW, Chou CX, Chang HF, Wu WC, Hsieh YW, Chen PC, Yu MY, Chang CC, Lin JR. Cognitive effects of weight-shifting controlled exergames in patients with chronic stroke: a pilot randomized comparison trial. Eur J Phys Rehabil Med. 2017 Oct;53(5):694-702.

24. Jordan K, Sampson M, King M. Gravity-Supported Exercise With Computer Gaming Improves Arm Function in Chronic Stroke. Arch Phys Med Rehabil [Internet]. 2014; Available from: http://dx.doi.org/10.1016/j.apmr.2014.02.028

25. Shin JH, Ryu H, Jang SH. A task-specific interactive game-based virtual reality rehabilitation system for patients with stroke: a usability test and two clinical experiments. J Neuroeng Rehabil. 2014 Dec 1;11(1):32.

26. Cameirão MS, i Badia SB, Oller ED, Verschure PF. Neurorehabilitation using the virtual reality based Rehabilitation Gaming System: methodology, design, psychometrics, usability and validation. J Neuroeng Rehabil. 2010 Dec 1;7(1):48.

27 Orihuela-Espina F, Fernandez del Castillo I, Palafox L, Pasaye E, Sánchez-Villavicencio I, Leder R, Hernández Franco J, Enrique Sucar L. Neural reorganization accompanying upper limb motor rehabilitation from stroke with virtual reality-based gesture therapy. Top Stroke Rehabil. 2013 May 1;20(3):197-209.

28. Gamito P, Oliveira J, Coelho C, Morais D, Lopes P, Pacheco J, Brito R, Soares F, Santos N, Barata AF. Cognitive training on stroke patients via virtual reality-based serious games. Disabil Rehabil. 2017 Feb 13;39(4):385-8.

29. Gauthier LV, Kane C, Borstad A, Strahl N, Uswatte G, Taub E, Morris D, Hall A, Arakelian M, Mark V. Video Game Rehabilitation for Outpatient Stroke (VIGoROUS): protocol for a multi-center comparative effectiveness trial of in-home gamified constraintinduced movement therapy for rehabilitation of chronic upper extremity hemiparesis. BMC Neurol. 2017 Dec;17(1):1-8.

30. Choi HS, Shin WS, Bang DH, Choi SJ. Effects of game-based constraint-induced movement therapy on balance in patients with stroke: a single-blind randomized controlled trial. Am J Phys Med Rehabil. 2017 Mar 1;96(3):184-90.

31. Sin H, Lee G. Additional virtual reality training using Xbox Kinect in stroke survivors with hemiplegia. Am J Phys Med Rehabil. 2013 Oct $1 ; 92(10): 871-80$

32. Singh DK, Nordin NA, Abd Aziz NA, Lim BK, Soh LC. Effects of substituting a portion of standard physiotherapy time with virtual reality games among community-dwelling stroke survivors. BMC neurol. 2013 Dec;13(1):1-7.

33. Bailey MJ, Riddoch MJ, Crome P. Treatment of visual neglect in elderly patients with stroke: a single-subject series using either a scanning and cueing strategy or a left-limb activation strategy. Phys Ther. 2002 Aug 1;82(8):782-97.

34. Wood SR, Murillo N, Bach-y-Rita P, Leder RS, Marks JT, Page SJ. Motivating, game-based stroke rehabilitation: a brief report. Top Stroke Rehabil. 2003 Jul 1;10(2):134-40.

35. Broeren J, Rydmark M, Sunnerhagen KS. Virtual reality and haptics as a training device for movement rehabilitation after stroke: a single-case study. Arch Phys Med Rehabil. 2004 Aug 1;85(8):1247-50.

36. Flynn S, Palma P, Bender A. Feasibility of using the Sony PlayStation 2 gaming platform for an individual poststroke: a case report. J Neurol Phys Ther. 2007 Dec 1;31(4):180-9.
37. Santos MC. Biorretroalimentación y juegos de computadora como complemento del manejo de limitación inducida sobre el control motor de la extremidad superior del paciente con secuelas de enfermedad vascular cerebral. Arch Neurocien. 2007;12(2):77-85.

38. Broeren J, Bjorkdahl A, Claesson L, Goude D, Lundgren-Nilsson A, Samuelsson H, Blomstrand C, Sunnerhagen KS, Rydmark M. Virtual rehabilitation after stroke. Stud Health Technol Inform. 2008 May;136:77.

39. Broeren J, Claesson L, Goude D, Rydmark M, Sunnerhagen KS. Virtual rehabilitation in an activity centre for community-dwelling persons with stroke. Cerebrovasc Dis. 2008;26(3):289-96.

40. Rand D, Kizony R, Weiss PT. The Sony PlayStation II EyeToy: lowcost virtual reality for use in rehabilitation. J Neurol Phys Ther. 2008 Dec 1;32(4):155-63.

41. Szturm T, Peters JF, Otto C, Kapadia N, Desai A. Task-specific rehabilitation of finger-hand function using interactive computer gaming. Arch Phys Med Rehabil. 2008 Nov 1;89(11):2213-7.

42. Yavuzer G, Senel A, Atay MB, Stam HJ. Playstation eyetoy games" improve upper extremity-related motor functioning in subacute stroke: a randomized controlled clinical trial. Eur J Phys Rehabil Med. 2008 Sep 1;44(3):237-44.

43. Lange B, Flynn S, Rizzo A. Initial usability assessment of off-theshelf video game consoles for clinical game-based motor rehabilitation. Phys Ther Rev. 2009 Oct 1;14(5):355-63.

44. Qiu Q, Fluet GG, Lafond I, Merians AS, Adamovich SV. Coordination changes demonstrated by subjects with hemiparesis performing hand-arm training using the NJIT-RAVR robotically assisted virtual rehabilitation system. In2009 Annual International Conference of the IEEE Engineering in Medicine and Biology Society 2009 Sep 3 (pp. 1143-1146). IEEE.

45. Ruparel R, Johnson MJ, Strachota E, McGuire J, Tchekanov G. Evaluation of the theradrive system for robot/computer assisted motivating rehabilitation after stroke. In2009 Annual International Conference of the IEEE Engineering in Medicine and Biology Society 2009 Sep 3 (pp. 811-814). IEEE. .

46. Golden S, Slezak K. Making things possible. Technology plays a role in neurorehabilitation for stroke patients. Rehab management. 2010;23(10):10-2.

47. Lange B, Flynn S, Proffitt R, Chang CY, "Skip" Rizzo A. Development of an interactive game-based rehabilitation tool for dynamic balance training. Top Stroke Rehabil. 2010 Sep 1;17(5):34552.

48. Saposnik G, Teasell R, Mamdani M, Hall J, Mcllroy W, Cheung D, Thorpe KE, Cohen LG, Bayley M. Effectiveness of virtual reality using Wii gaming technology in stroke rehabilitation: a pilot randomized clinical trial and proof of principle. Stroke. $2010 \mathrm{Jul}$ 1;41(7):1477-84. 49. Joo LY, Yin TS, Xu D, Thia E, Chia PF, Kuah CW, He KK. A feasibility study using interactive commercial off-the-shelf computer gaming in upper limb rehabilitation in patients after stroke. J Rehabil Med. 2010 May 1;42(5):437-41.

50. Cameirão MS, Badia SB, Duarte E, Verschure PF. Virtual reality based rehabilitation speeds up functional recovery of the upper extremities after stroke: a randomized controlled pilot study in the acute phase of stroke using the rehabilitation gaming system. Restor Neurol Neurosci. 2011;29(5):287-98.

51. Hijmans JM, Hale LA, Satherley JA, MCMillan NJ, King MJ. Bilateral upper-limb rehabilitation after stroke using a movement-based game controller. J Rehabil Res Dev. 2011 Nov 1;48(8):1005-14.

52. Merians AS, Fluet GG, Qiu Q, Saleh S, Lafond I, Davidow A, Adamovich SV. Robotically facilitated virtual rehabilitation of arm transport integrated with finger movement in persons with hemiparesis. J Neuroeng Rehabil. 2011 Dec 1;8(1):27.

53. Mouawad MR, Doust CG, Max MD, McNulty PA. Wii-based movement therapy to promote improved upper extremity function post-stroke: a pilot study. J Rehabil Med. 2011 May 5;43(6):527-33.

54. Schuck SO, Whetstone A, Hill V, Levine P, Page SJ. Game-based, portable, upper extremity rehabilitation in chronic stroke. Top Stroke Rehabil. 2011 Nov 1;18(6):720-7.

55. Acosta AM, Dewald HA DJ. Pilot study to test effectiveness of video game on reaching. J Rehabil Res Dev. 2012;48(4):431-44.

56. Combs SA, Finley MA, Henss M, Himmler S, Lapota K, Stillwell D. Effects of a repetitive gaming intervention on upper extremity impairments and function in persons with chronic stroke: a preliminary study. Dis Rehabil. 2012 Jul 1;34(15):1291-8. 
57. Kim EK, Kang JH, Park JS, Jung BH. Clinical feasibility of interactive commercial Nintendo gaming for chronic stroke rehabilitation. J Phys Ther Sci. 2012;24(9):901-3.

58. Hale LA, Satherley JA, MCMillan NJ, Milosavljevic S, Hijmans JM, King MJ. Participant perceptions of use of CyWee $Z$ as adjunct to rehabilitation of upper-limb function following stroke. J Rehabil Res Dev. 2012 Jul 1;49(4).

59. Kam N, Struzik J, Jarus T, Rand D. Is the Nintendo Wii suitable for stroke rehabilitation? A pilot feasibility and usability study. Israeli $J$ Occup Ther. 2012 Feb;21(1):3-25.

60. Kim I. Effects of an enjoyable nurse-led intervention to promote movement in poststroke inpatients. Clin Nurs Res. 2012 Nov;21(4):390-405.

61. King M, Hijmans JM, Sampson M, Satherley J, Hale L. Home-based stroke rehabilitation using computer gaming. New Zealand J. Phys. 2012 Nov 1;40(3)

62. Reinthal A, Szirony K, Clark C, Swiers J, Kellicker M, Linder S. ENGAGE : Guided Activity-Based Gaming in Neurorehabilitation after Stroke : A Pilot Study. Stroke Res Treat. 2012;2012. doi: $10.1155 / 2012 / 784232$

63. Taheri H, Rowe JB, Gardner D, Chan V, Reinkensmeyer DJ, Wolbrecht ET. Robot-assisted guitar hero for finger rehabilitation after stroke. In2012 Annual International Conference of the IEEE Engineering in Medicine and Biology Society 2012 Aug 28 (pp. 3911. 3917). IEEE.

64. Finley M, Combs S. User perceptions of gaming interventions for improving upper extremity motor function in persons with chronic stroke. Physiother Theory Pract. 2013 Apr 1;29(3):195-201.

65. Fritz SL, Peters DM, Merlo AM, Donley J. Active video-gaming effects on balance and mobility in individuals with chronic stroke: a randomized controlled trial. Top Stroke Rehabil. 2013 May $1 ; 20(3): 218-25$.

66. Lee G. Effects of training using video games on the muscle strength, muscle tone, and activities of daily living of chronic stroke patients. J Phys Ther Sci. 2013 May 25;25(5):595-7.

67. Peters DM, MCPherson AK, Fletcher B, McClenaghan BA, Fritz SL. Counting repetitions: an observational study of video game play in people with chronic poststroke hemiparesis. J Neurol PhysTher. 2013 Sep 1;37(3):105-11.

68. Wüest S, Van De Langenberg R, De Bruin ED. Design considerations for a theory-driven exergame-based rehabilitation program to improve walking of persons with stroke. European Review of Aging and Physical Activity. 2014 Oct 1;11(2):119-29.

Simmons CD, Arthanat S, Macri VJ. Pilot study: Computer-based virtual anatomical interactivity for rehabilitation of individuals with chronic acquired brain injury. J Rehabil Res Dev. 2014 Mar 1;51(3).

70 Goodman RN, Rietschel JC, Roy A, Jung BC, Diaz J, Macko RF, Forrester LW. Increased reward in ankle robotics training enhances motor control and cortical efficiency in stroke. J Rehabil Res Dev. 2014 Feb 1;51(2).

71. Bower KJ, Clark RA, McGinley JL, Martin CL, Miller KJ. Clinical feasibility of the Nintendo $\mathrm{Wii}^{\mathrm{M}}$ for balance training post-stroke: a phase II randomized controlled trial in an inpatient setting. Clin Rehabil. 2014 Sep;28(9):912-23.

72. Brown EVD, Otr L, Mccoy SW, Fechko AS, Price R, Gilbertson T, et al. Preliminary Investigation of an Electromyography- Controlled Video Game as a Home Program for Persons in the Chronic Phase of Stroke Recovery. Arch Phys Med Rehabil [Internet]. 2014; Available from: http://dx.doi.org/10.1016/j.apmr.2014.02.025

73. Friedman N, Chan V, Reinkensmeyer AN, Beroukhim A, Zambrano GJ, Bachman M, Reinkensmeyer DJ. Retraining and assessing hand movement after stroke using the MusicGlove: comparison with conventional hand therapy and isometric grip training. J Neuroeng Rehabil. 2014 Dec;11(1):1-4.

74. Hung J, Chou C, Hsieh Y, Wu W, Yu M, Chen P, et al. A Randomized Comparison Trial of Balance Training by Using Exergaming and Conventional Weight-Shifting Therapy in Patients with Chronic Stroke. Arch Phys Med Rehabil [Internet]. 2014; Available from: http://dx.doi.org/10.1016/j.apmr.2014.04.029

75. Kafri M, Myslinski MJ, Gade VK, Deutsch JE. Energy expenditure and exercise intensity of interactive video gaming in individuals poststroke. Neurorehabil Neural Repair. 2014 Jan;28(1):56-65.

76. Morone G, Tramontano M, losa M, Shofany J, lemma A, Musicco $M$, et al. The Efficacy of Balance Training with Video Game-Based
Therapy in Subacute Stroke Patients : A Randomized Controlled Trial. Biomed Res Int. 2014;2014:1-6.

77. Novak D, Nagle A, Keller U, Riener R. Increasing motivation in robot-aided arm rehabilitation with competitive and cooperative gameplay. J Neuroeng Rehabil. 2014 Dec;11(1):64.(1-15).

78. Pompeu JE, Alonso TH, Masson IB, Maria S, Pompeu AA, Torrianipasin C. Os efeitos da realidade virtual na reabilitação do acidente vascular encefálico : Uma revisão sistemática The effects of virtual reality on stroke rehabilitation : A systematic review. Motricidade. 2014;10(4):111-22.

79. Shiner CT, Byblow WD, McNulty PA. Bilateral priming before Wiibased movement therapy enhances upper limb rehabilitation and its retention after stroke: a case-controlled study. Neurorehabil Neural Repair. 2014 Nov;28(9):828-38.

80. Subramaniam S, Hui-Chan CW, Bhatt T. A cognitive-balance control training paradigm using wii fit to reduce fall risk in chronic stroke survivors. J Neurol Phys Ther. 2014 Oct 1;38(4):216-25.

81. Taheri H, Rowe JB, Gardner D, Chan V, Gray K, Bower C, Reinkensmeyer DJ, Wolbrecht ET. Design and preliminary evaluation of the FINGER rehabilitation robot: controlling challenge and quantifying finger individuation during musical computer game play. J Neuroeng Rehabil. 2014 Dec 1;11(1):10.

82. Viana RT, Fonseca JB, Filho EMS, Dias SN. Effects of the addition of transcranial direct current stimulation to virtual reality therapy after stroke : A pilot randomized controlled trial. NeuroRehabil. 2014;34:437-46.

83. Chen MH, Huang LL, Lee CF, Hsieh CL, Lin YC, Liu H, Chen MI, Lu WS. A controlled pilot trial of two commercial video games for rehabilitation of arm function after stroke. Clin Rehabil. 2015 Jul;29(7):674-82.

84. Tsekleves E, Paraskevopoulos IT, Warland A, Kilbride C. Development and preliminary evaluation of a novel low cost VR-based upper limb stroke rehabilitation platform using Wii technology. Disabil Rehabil Assist Technol. 2016 Jul 3;11(5):413-22.

85. Wingham J, Adie K, Turner D, Schofield C, Pritchard C. Participant and caregiver experience of the Nintendo Wii SportsTM after stroke: qualitative study of the trial of WiiTM in stroke (TWIST). Clin Rehabi. 2015 Mar;29(3):295-305.

86. Omiyale O, Crowell CR, Madhavan S. Effect of Wii-based balance training on corticomotor excitability post stroke. J Mot Behav. 2015 May 4;47(3):190-200.

87. Anderson KR, Otr L, Woodbury ML, Otr L, Phillips K, Gauthier L V. Information / Education Page Virtual Reality Video Games to Promote Movement Recovery in Stroke Rehabilitation : A Guide for Clinicians. Arch Phys Med Rehabil [Internet]. 2015;96(5):973-6.

88. Bower KJ, Louie J, Landesrocha Y, Seedy P, Gorelik A, Bernhardt J. Clinical feasibility of interactive motion- controlled games for stroke rehabilitation. J Neuroeng Rehabil [Internet]. 2015;1-12.

89. da Silva Ribeiro NM, Ferraz DD, Pedreira É, Pinheiro Í, da Silva Pinto AC, Neto MG, Dos Santos LR, Pozzato MG, Pinho RS, Masruha MR. Virtual rehabilitation via Nintendo $\mathrm{Wii} \odot$ and conventional physical therapy effectively treat post-stroke hemiparetic patients. Top Stroke Rehabil. 2015 Aug 1;22(4):299-305.

90. Brown EVD, Dudgeon BJ, Gutman K, Chet T. Understanding Upper Extremity Home Programs and the use of Gaming Technology for Persons After Stroke. Disabil Health J [Internet]. 2015; Available from: http://dx.doi.org/10.1016/j.dhjo.2015.03.007

91. Lee HY, Kim YL, Lee SM. Effects of virtual reality-based training and task-oriented training on balance performance in stroke patients. J Phys Ther Sci. 2015;27(6):1883-8.

92Lee KH. Effects of a virtual reality-based exercise program on functional recovery in stroke patients: part 1. J Phys Ther Sci. 2015;27(6):1637-40.

93. Macdonald JA, Gauthier L. Research Posters. Arch Phys Med Rehabil [Internet]. 96(12):e6-7. Available from: http://dx.doi.org/10.1016/j.apmr.2015.10.008

94. McNulty PA, Thompson-Butel AG, Faux SG, Lin G, Katrak PH, Harris LR, Shiner CT. The efficacy of Wii-based Movement Therapy for upper limb rehabilitation in the chronic poststroke period: a randomized controlled trial. Int J Stroke. 2015 Dec;10(8):1253-60.

95. Mobini A, Behzadipour S, Saadat M. Test-retest reliability of Kinect's measurements for the evaluation of upper body recovery of stroke patients. Biomed Eng Online. 2015 Dec 1;14(1):75.

96. Ponte S, Gabrielli S, Jonsdottir J, Morando M, Dellepiane S. Monitoring game-based motor rehabilitation of patients at home for 
better plans of care and quality of life. In 2015 37th Annual International Conference of the IEEE Engineering in Medicine and Biology Society (EMBC) 2015 Aug 25 (pp. 3941-3944). IEEE.

97. P Prange GB, Kottink Al, Buurke JH, Eckhardt MM, van KeulenRouweler BJ, Ribbers GM, Rietman JS. The effect of arm support combined with rehabilitation games on upper-extremity function in subacute stroke: a randomized controlled trial. Neurorehabil Neural Repair. 2015 Feb;29(2):174-82.

98. Buick AR, Kowalczewski J, Carson RG PA. Tele Supervised FESexercise hemiplegic upper limb. IEEE Trans Neural Syst Rehabil Eng. 2016 Jan;24(1):79-87.

99. Givon N, Zeilig G, Weingarden H, Rand D. Video-games used in a group setting is feasible and effective to improve indicators of physical activity in individuals with chronic stroke: a randomized controlled trial. Clin Rehabil. 2016 Apr;30(4):383-92.

100. Llorens R, Latorre J, Noé E, Keshner EA. Posturography using the Wii Balance Board ${ }^{\mathrm{m}}$ : a feasibility study with healthy adults and adults post-stroke. Gait Posture. 2016 Jan 1;43:228-32.

101. Bonuzzi GM, Freitas TB, Corrêa UC, Freudenheim AM, Pompeu $\mathrm{JE}$, Torriani-Pasin C. Learning of a postural control task by elderly post-stroke patients. Motricidade. 2016;12(1):141-8.

102. Shin J, Kim M, Lee J, Jeon Y, Kim S, Lee S, et al. Effects of virtual reality-based rehabilitation on distal upper extremity function and health-related quality of life : a single- blinded, randomized controlled trial. J Neuroeng Rehabil [Internet]. 2016;1-10.

103. Kong K, Loh $\mathrm{Y}$, Thia E, Chai A, Ng C, Soh M, et al. Efficacy of a Virtual Reality Commercial Gaming Device in Upper Limb Recovery after Stroke : A Randomized, Controlled Study Efficacy of a Virtual Reality Commercial Gaming Device in Upper Limb Recovery after Stroke : A Randomized, Controlled Study. Top Stroke Rehabil [Internet]. 2016;9357:1-8.

104. Lee M, Pyun SB, Chung J, Kim J, Eun SD, Yoon B. A further step to develop patient-friendly implementation strategies for virtual reality-based rehabilitation in patients with acute stroke. Phys Ther. 2016 Oct 1;96(10):1554-64.

105. Mousavi Hondori H, Khademi M, Dodakian L, McKenzie A, Lopes $\mathrm{CV}$, Cramer SC. Choice of human-computer interaction mode in stroke rehabilitation. Neurorehabil Neural Repair. 2016 Mar;30(3):258-65.

106. Paquin K, Crawley J, Harris JE, Horton S. Survivors of chronic stroke-participant evaluations of commercial gaming for rehabilitation. Dis Rehabil. 2016 Oct 8;38(21):2144-52.

107. Proffitt R, Henderson W. LSVT-BIG in Stroke: A Case Study. Arch Phys Med Rehabil [Internet]. 2016;97(12):e17.

108. Rinne P, Mace M, Nakornchai T, Zimmerman K, Fayer S, Sharma P, Liardon JL, Burdet E, Bentley P. Democratizing neurorehabilitation: How accessible are low-cost mobile-gaming technologies for self-rehabilitation of arm disability in stroke?. PloS one. 2016 Oct 5;11(10):e0163413.

109. Saposnik G, Cohen LG, Mamdani M, Pooyania S, Ploughman M, Cheung D, et al. Effi cacy and safety of non-immersive virtual reality exercising in stroke rehabilitation ( EVREST ): a randomised, multicentre, single-blind, controlled trial. Lancet Glob Heal [Internet]. 2016;4422(16):1-9.

110. Șimșek $\Pi$, Çekok K. The effects of Nintendo WiiTM-based balance and upper extremity training on activities of daily living and quality of life in patients with sub-acute stroke: a randomized controlled study. Int J Neurosci. 2016 Dec 1;126(12):1061-70.

111. Bang YS, Son KH, Kim HJ. Effects of virtual reality training using Nintendo Wii and treadmill walking exercise on balance and walking for stroke patients. J Phys Ther Sci. 2016;28(11):3112-5.

112. Zondervan DK, Friedman N, Chang E, Zhao X, Augsburger R, Reinkensmeyer DJ, Cramer SC. Home-based hand rehabilitation after chronic stroke: Randomized, controlled single-blind trial comparing the MusicGlove with a conventional exercise program. J Rehabil Res Dev. 2016;53(4):457-72.

113. Nijenhuis SM, Prange-Lasonder GB, Stienen AH, Rietman JS, Buurke JH. Effects of training with a passive hand orthosis and games at home in chronic stroke: a pilot randomised controlled trial. Clin rehabil. 2017 Feb;31(2):207-16.;

114. Rand D, Weingarden H, Weiss R, Yacoby A, Reif S, Malka R, et al. Self-training to improve UE function at the chronic stage poststroke : a pilot randomized controlled trial. Disabil Rehabil [Internet]. 2017 Jul 17;39(15):1541-8.

115. Standen PJ, Threapleton K, Connell L, Richardson A, Brown DJ, Battersby S, Sutton CJ, Platts F. Patients' use of a home-based virtual reality system to provide rehabilitation of the upper limb following stroke. Phys Ther. 2015 Mar 1;95(3):350-9.

116. Trinh T, Shiner CT, Thompson-butel AG, Penelope A, Trinh T, Shiner CT, et al. Targeted upper-limb Wii-based Movement Therapy also improves lower-limb muscle activation and functional movement in chronic stroke. Disabil Rehabil. 2017 Sep 11;39(19):1939-49.

117. Bouchard AE, Corriveau H, Milot MH. A single robotic session that guides or increases movement error in survivors post-chronic stroke: which intervention is best to boost the learning of a timing task? Disabil Rehabil. 2017 Jul 31;39(16):1607-14.

118. Grossi M, Fabrin S, Soares N, Regalo SC, Zanella CA, Verri ED. Benefícios do video game em pacientes com sequelas de acidente vascular cerebral. Fisioterapia Brasil. 2017 Jun 25;18(3):356-61.

119. James TT. Effect of Gaming Assisted Visual Feedback on Functional Standing Balance among Acute Hemiparetic Stroke Patients. Indian J Physiother OccupTher. 2017 Oct 1;11(4).

120. Park D, Lee D, Lee K, Lee G. Effects of Virtual Reality Training using Xbox Kinect on Motor Function in Stroke Survivors : A Preliminary Study. J Stroke Cerebrovasc Dis. 2017;1-7.

121. Rowe JB, Chan V, Ingemanson ML, Cramer SC, Wolbrecht ET, Reinkensmeyer DJ. Robotic assistance for training finger movement using a hebbian model: a randomized controlled trial. Neurorehabil Neural Repair. 2017 Aug;31(8):769-80.;

122. Jung SM, Choi WH. Effects of virtual reality intervention on upper limb motor function and activity of daily living in patients with lesions in different regions of the brain. J Phys Ther Sci. 2017;29(12):2103-6.

123. Carregosa AA, Rafael L, Masruha MR, Lira M, Coêlho S, Machado TC, et al. Virtual Rehabilitation through Nintendo Wii in Poststroke Patients : Follow-Up. J Stroke Cerebrovasc Dis. 2017;1-5.

124. Choi YH, Paik NJ. Mobile game-based virtual reality program for upper extremity stroke rehabilitation. JoVE (Journal of Visualized Experiments). 2018 Mar 8(133):e56241.

125. Cesar J, Sousa S De, Torriani-pasin C, Tosi AB, Fecchio RY, Augusto $\mathrm{L}$. Aerobic stimulus induced by virtual reality games in stroke survivors. Arch Phys Med Rehabil [Internet]. 2018; Available from: https://doi.org/10.1016/j.apmr.2018.01.014 